\title{
EL GÉNERO OROBANCHE L. (OROBANCHACEAE) EN ANDALUCÍA
}

\author{
Antonio J. PUJADAS SALVÀ ${ }^{1 *}$, Laura PLAZA ARREGUI ${ }^{1}$, Enrique SÁNCHEZ \\ GULLÓN $^{2}$, Enrique TRIANO MUÑOZ ${ }^{3}$, Mónica LÓPEZ MARTÍNEZ ${ }^{4}$, Concetta \\ BURGARELLA ${ }^{1}$, Diego RUBIALES OLMEDO ${ }^{5}$, Belén ROMÁN DEL CASTILLO 6 , \\ Emilio REYES SEPÚLVEDA ${ }^{7} \&$ Andrés IVORRA JIMÉNEZ ${ }^{8}$
}

\footnotetext{
${ }^{1}$ Departamento de Ciencias y Recursos Agrícolas y Forestales. Edificio Celestino Mutis. Campus de Rabanales. Universidad de Córdoba. E-14071 Córdoba.

${ }^{2}$ Paraje Natural Marismas del Odiel. Ctra. del Dique Juan Carlos I. Apdo. 720. E-21071 Huelva.

${ }^{3}$ Digitalis Ibérica S. L. C/ Redondo Marqués 12, 20. E-14940 Cabra, Córdoba.

${ }^{4}$ Jardín Botánico de Córdoba. Avda. Linneo s/n. E-14004 Córdoba.

${ }^{5}$ Instituto de Agricultura Sostenible, CSIC. Apdo. 4084. E-14080 Córdoba.

${ }^{6}$ Departamento de Mejora y Agronomía. CIFA. Alameda del Obispo. Apdo 3092. E-14080 Córdoba.

${ }^{7}$ C/ María La Judía 10, 2º-1 D. 14011 Córdoba.

${ }^{8} \mathrm{C} /$ Tiendas 34. E-04003 Almería.

*Autor para correspondencia: crlpusaa@uco.es
}

Recibido el 12 de marzo de 2007, aceptado para su publicación el 10 de julio de 2007 Publicado "on line" en octubre de 2007

RESUMEN. El género Orobanche L. (Orobanchaceae) en Andalucía. Se actualiza el conocimiento del género Orobanche L. para la Flora de Andalucía, sur de la Península Ibérica. La revisión se ha hecho mediante el análisis crítico de la información bibliográfica y de los principales herbarios relacionados con el territorio. Para facilitar la identificación de las diferentes especies aportamos una clave de determinación. Para los 31 taxones presentes aportamos su distribución detallada en unidades geográficas así como su categoría UICN. Resaltamos la presencia de taxones de marcado interés corológico, poco citados en todo el territorio, como O. ramosa, O. olbiensis, O. mariana, O. lavandulacea, O. schultzii, O. mutelii subsp. georgii-reuteri, O. purpurea, O. clausonis, O. rapumgenistae, O. gracilis subsp. deludens, O. haenseleri, $O$. alba, O. icterica, O. amethystea subsp. castellana, O. almeriensis, $O$. santolinae. Consideramos que especies como O. sanguinea, $O$. crinita, $O$. variegata, $O$. caryophyllaceae, $O$. lutea, $O$. reticulata y $O$. artemisiae-campestris no se encuentran en Andalucía y a $O$. calendulae como especie conflictiva a buscar.

Palabras clave. Orobanche, Orobanchaceae, jopo, parásita, corología, Andalucía.

ABSTRACT. The genus Orobanche (Orobanchaceae) in Andalusia. A botanical survey has been carried out in order to improve the knowledge of the genus Orobanche L. of the Flora of Andalusia, South of the Iberian Peninsula. With this aim, a critical review of the concerning bibliographical information and of the main territorial herbaria was accomplished. An identification key is provided. For each of the 31 taxa considered its detailed distribution in geographical units as well as its category UICN are given. The presence of taxa of important chorological interest such as $O$. ramosa, O. olbiensis, $O$. mariana, O. lavandulacea, O. schultzii, O. mutelii subsp. georgii-reuteri, O. purpurea, O. clausonis, $O$. rapum-genistae, $O$. gracilis subsp. deludens, $O$. haenseleri, $O$. alba, $O$. icterica, $O$. amethystea subsp. castellana, $O$. almerienses, $O$. santolinae is pointed out. Moreover, we consider that $O$. 
sanguinea, O. crinita, O. variegata, O. caryophyllaceae, O. lutea, O. reticulata and $O$. artemisiaecampestris are species not found in Andalusia and $O$. calendulae is a conflictive taxon to investigate.

Key words. Orobanche, Orobanchaceae, broomrape, parasite, chorology, Andalusia.

\section{INTRODUCCIÓN}

El estudio detallado del género Orobanche en la Comunidad Autónoma de Andalucía se inició con nuestros trabajos sobre la sect. Trionychon (Pujadas Salvà, 2000; Pujadas Salvà et al., 2005) y con las diferentes aportaciones provinciales (Pujadas Salvà \& Lora González, 1996; Molino Olmedo et al., 1995) o aportaciones parciales sobre algunas especies conflictivas (Pujadas Salvà et al., 1994; Pujadas \& Lora, 1995; Pujadas Salvà \& Lora González, 1997; Pujadas Salvà, 1997; Pujadas Salvà \& Velasco, 2000; Pujadas Salvà et al., 2003; Pujadas Salvà \& Plaza Arregui, 2004; Pujadas Salvà et al., 2005). Por otra parte, la preparación de la redacción de la familia Orobanchaceae para la Flora de Andalucía Oriental y para la Flora Bético Rifeña ha supuesto la actualización sobre su conocimiento, que presentamos aquí.

Completamos, en primer lugar, la información disponible sobre la corología de las especies del subgénero Phelipanche (Pomel) Tzevelev (sect. Trionychon), ya estudiada en Pujadas Salvà (2000), y a continuación aportamos la de las especies correspondientes a los subgéneros Boulardia (F.W. Schltuz) A. Pujadas y Orobanche L. (sect. Orobanche).

\section{MATERIAL Y MÉTODOS}

Seguimos el procedimiento indicado en Pujadas Salvà (2000) y Pujadas Salvà et al. (2005) ampliado con el estudio total o parcial de los siguientes herbarios: ARAN, CGE, COI, SÁNCHEZ PEDRAJA, HUAL, LEB,
MANCH, PRC, SALA/SALAF, VAL, W, WU. Añadimos solamente aquellas sinonimias que no aparecen en Pujadas Salvà (2000; 2002). La iconografía, descripción detallada, hábitat, hospedantes y corología corresponden a las publicadas en Pujadas Salvà $(2000 ; 2002)$ y a las de la Flora de Andalucía Oriental y de la Flora Bético Rifeña que están en prensa. Para la sectorización de Andalucía adoptamos las unidades territoriales descritas por Valdés (2005) (fig. 1).

Aportamos un máximo de cinco referencias de herbario para cada unidad geográfica y provincia - para evitar que el trabajo sea demasiado extenso-con las excepciones de $O$. rapum-genistae Thuill., $O$. haenseleri Reut. y $O$. santolinae Loscos \& J. Pardo para los que, por su interés corológico, se indican todas las localidades estudiadas. En todos los casos se ha intentado evitar repeticiones de una misma localidad.

Siempre que ha sido posible hemos contrastado los testimonios de herbario de las referencias bibliográficas existentes, principalmente las de Willkomm (1870), Willkomm (1893), Cuatrecasas (1929), Molero Mesa \& Pérez Raya (1987), Valdés \& Talavera (1991), Molino Olmedo et al. (1995), Foley (2001b). Análogamente señalamos las enmiendas en Pujadas Salvà \& Lora González (1996).

Proponemos las categorías UICN (2001) a nivel regional para cada taxón considerando a las Orobanchaceae (holoparásitos) como taxones independientes a pesar de que en UICN (2001, Apdo. a nivel regional III, Def. 1) se indica que "En el caso de los taxones que dependen obligatoriamente de otro taxón en todo o en parte de su ciclo de vida, deben de 


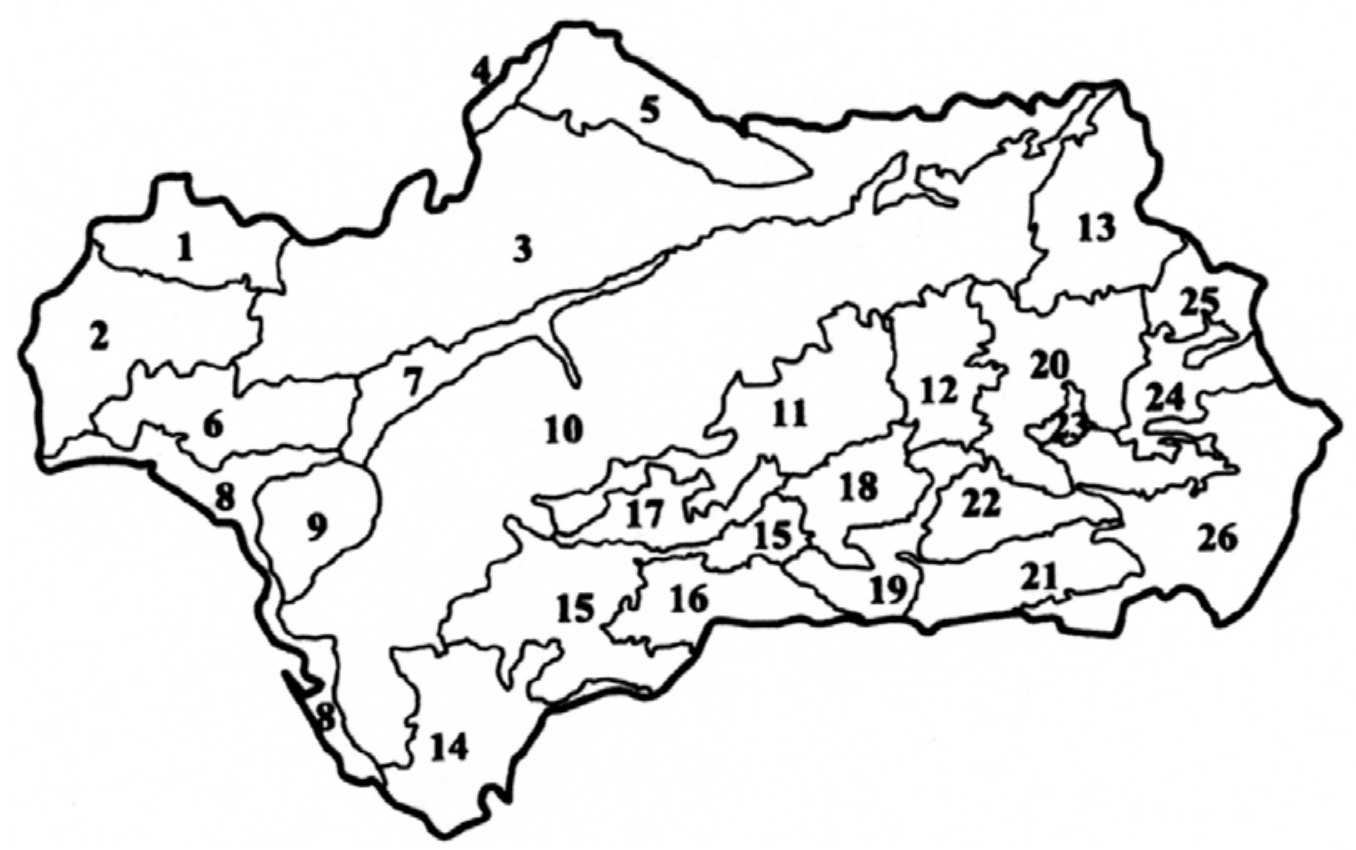

Figura 1. Unidades geográficas de Andalucía (Valdés, 2005). 1. Sierra de Aracena. 2. Andévalo. 3. Sierra Morena. 4. Zújar. 5. Pedroches. 6. Campiña de Huelva y Condado-Aljarafe. 7. Vega del Guadalquivir. 8. Litoral. 9. Marismas del Guadalquivir. 10. Valle del Guadalquivir. 11. Subbética Occidental. 12. Sierra Mágina. 13. Cazorla-Segura-Las Villas. 14. Sierra del Aljibe. 15. Serranía de Ronda. 16. Axarquía. 17. Depresión de Antequera. 18. Depresión de Granada. 19. Sierras de Almijara, Guájares y Trevenque. 20. Depresión de Guadix-Baza y Guadiana Menor. 21. Alpujarras bajas, Contraviesa y Sierra de Gádor. 22. Sierra Nevada y Filabres. 23. Sierra de Baza. 24. Sierras de María, Orce y Estancias. 25. Altiplanos Manchegos y Almería Subbética. 26. Almería Subdesértica. Geographical units of Andalusia (Valdés, 2005).

usarse los valores apropiados para el taxón hospedador."

\section{RESULTADOS}

OROBANCHE L., Sp. Pl. 632 (1753) [Gen. $P l .$, ed. 5, 281, 1754]

1. Flores con dos bractéolas y una bráctea. Plantas de tallo ramificado o simple ......... 2

1. Flores sólo con una bráctea. Plantas de tallo simple no ramificado. 10

2. Labio inferior de la corola con lóbulos obovados u oblongos, obtusos de ápice $+/-$ redondeados, margen cortamente dentado 3
2. Labio inferior de la corola con lóbulos elípticos, ovados o lanceolados, agudos, acuminados o si el ápice es subagudo el margen es profundamente dentado ............ 8

3. Cáliz (13)16-21 mm. Corola de (18)21-30 $\mathrm{mm}$, purpúrea o azulada ....... 9. O. arenaria

3. Cáliz 4-12 mm. Corola de 12-25 mm, blanquecina a azul intenso en el ápice ...... 4

4. Cáliz 4-7,5 mm. Corola 12-16(17) mm ..... 5

4. Cáliz 8-12 mm. Corolla 17-25 mm ........... 6

5. Planta $17-35 \mathrm{~cm}$, tallo muy ramificado desde la base (por debajo del nivel del suelo). Inflorescencia 10-25 cm, laxa. Dientes del cáliz triangulares brevemente acuminados. Corola blanquecina o azul pálido en el ápice 1. O. ramosa 
5. Planta 8-20 (27) cm, tallo simple o ramificado. Inflorescencia 3,5-7(8) $\mathrm{cm}$, densa a veces laxa en la base. Dientes del cáliz lanceolados a triangulares, largamente acuminados de ápice subfiliforme. Corola azul en el ápice, a veces intensamente azulmorado

2. O. nana

6. Planta densa y uniformemente pubescente glandular. Tallo simple. Corola azul o morado pálido con venas longitudinales conspicuas más oscuras

8. O. purpurea

6. Planta pubescente, vilosa o cortamente aracnoidea glandular. Tallo simple o ramificado. Corola azul oscuro o azul pálido, sin venas longitudinales conspicuas más oscuras

7. Planta (25)33-53 cm, frecuentemente con ramificaciones basales cortas portadoras de yemas floríferas estériles. Inflorescencia (12)19-47 cm. Corola azul oscuro intenso ...

6. O. lavandulacea

7. Planta (8)10-28 cm, sin ramificaciones basales cortas portadoras de yemas floríferas estériles. Inflorescencia $(2,5) 4-13 \mathrm{~cm}$. Corola de azul pálido a azul brillante en el ápice....

5. O. mutelii

8. Cáliz (10)13-19 mm, con dientes 1,5-3,5 veces la longitud del tubo. Corola (14)17-23 $\mathrm{mm}$

7. O. schultzii

8. Cáliz 4-12 mm, con dientes menores que 1,5 veces la longitud del tubo. Corola 12-17 mm

9. Inflorescencia 3-5 cm. Brácteas 5-7 $\mathrm{mm}$. Bractéolas 3,5-6 mm. Cáliz 6-9 mm, frecuentemente dientes ligeramente falcados. Margen de la corola cortamente ciliado con pelos glandulares de 0,2-0,3 $\mathrm{mm}$. Anteras vilosas con pelos de hasta $0,4 \mathrm{~mm}$ en su base 3. O. olbiensis

9. Inflorescencia 6-11 cm. Brácteas 7-10 mm. Bractéolas 5,5-7 mm. Cáliz 7-11 mm, dientes rectos. Margen de la corola glabro o glabrescente con pelos glandulares de 0,02$0,04 \mathrm{~mm}$. Anteras glabras ..... 4. O. mariana

10. Estigma blanco 11

10. Estigma amarillo, naranja, morado, rosado o rojo

11. Brácteas 16-29 x 7-14 mm, ampliamente ovadas. Cáliz (9)13-21 mm. Corola (21)25$35 \mathrm{~mm}$, marcadamente galeada

10. O. latisquama

11. Brácteas (5)7-17(19) x 3-7 mm, ovadas a lanceoladas. Cáliz 5-14 mm. Corola 10-22 $\mathrm{mm}$, no o escasamente galeada 12

12. Corola de limbo morado, azul, azul pálido, raramente blanca. Filamentos adaxiales insertos a (5)6-9 $\mathrm{mm}$ de la base de la corola

13

12. Corola de limbo completamente blanco o blanco u ocroleuco con las venas y el ápice teñido de morado. Filamentos adaxiales insertos a 2-4(5) $\mathrm{mm}$ de la base de la corola

13. Planta de 15-32 $\mathrm{cm}$. Inflorescencia (4)6$17(23) \mathrm{cm}$, densa a veces laxa en la base. Segmentos del cáliz bífidos a bipartidos, raramente enteros. Corola (13)15-18 mm, de limbo azul oscuro o morado . 11. O. cernua

13. Planta de (35)40-65 cm. Inflorescencia (15)22-30(38) cm, laxa a veces sólo densa en el ápice. Segmentos del cáliz enteros, a veces bífidos. Corola (16)19-22 mm, de limbo blanco o azul pálido ............ 12. O. cumana

14. Cáliz 8-14 mm. Corola 15-18(20) mm, dorso recto, raramente curvado, ocroleuca o blanca con venas y ápice teñido de color morado ...

24. O. santolinae

14. Cáliz 5-9 mm. Corola 10-14(15) mm, dorso curvado, color blanco lechoso, raramente con los nervios de los lóbulos ligeramente violáceos

26. O. ballotae

15. Estigma amarillo o naranja ....................... 16

15. Estigma morado, rosado o rojo ................ 29

16. Margen de la corola peloso glandular ..... 17

16. Margen de la corola glabro o raramente subglabro 21

17. Corola color rojo oscuro brillante, rojo sanguíneo interiormente....... 15. O. gracilis

17. Corola color amarillento, ocroleuco, anaranjado, pardo rojizo, cárneo claro, rosado, vinoso o raramente rojizo interiormente, pero no rojo sanguíneo interiormente

18. Filamentos adaxiales insertos a $(0,5) 1$ $2,5(3,5) \mathrm{mm}$ de la base de la corola, de base pubérula o glabra ........................................ 19

18. Filamentos adaxiales insertos a $2,5-6 \mathrm{~mm}$ de la base de la corola, de base densamente pelosa, raramente pubérula 20

19. Margen de la corola ciliado, a veces cortamente, con pelos glandulares de 0,2-0,5 $\mathrm{mm}$. Base de los filamentos pubérula, a veces glabrescente. Estigma bilobado

15. O. gracilis

19. Margen de la corola cortamente peloso, con 
escasos pelos glandulares de menos de 0,1 $\mathrm{mm}$. Base de los filamentos glabra. Estigma bifurcado.....

14. O. rapum-genistae

20. Cáliz 12-20 mm, igualando frecuentemente el tubo de la corola, segmentos libres, contiguos o separados, bífidos a cortamente bipartidos. Corola ampliamente tubular campanulada a ligeramente infundibuliforme. Anteras 2,4-2,9 mm ........ 18. O. haenseleri

20. Cáliz 8-11(15) mm, menor que el tubo de la corola, segmentos contiguos o superpuestos, raramente connatos, subenteros o bífidos. Corola tubular, más estrecha por debajo de la inserción de los filamentos, ligeramente infundibuliforme. Anteras 1,8-2,1(2,5) mm.

20. O. icterica

21. Corola tubular, hinchada en la base, estrechándose de forma atenuada por encima de la inserción de los filamentos.....

27. O. hederae

21. Corola ampliamente tubular, campanulada o infundibuliforme, sin estrecharse por encima de la inserción de los filamentos. 22

22. Corola color rojo oscuro brillante (color sangre) interiormente 16. O. foetida

22. Corola blanquecina, amarillenta, ocroleuca, anaranjada, pardo rojiza, cárnea clara, rosada, vinosa o raramente rojiza, pero no rojo sanguínea interiormente. 23

23. Corola de 11-22 mm. Anteras 1-1,7 mm 24 23. Corola 17-29 mm. Anteras 1,9-2,9(3) mm.. 26

24. Inflorescencia muy densa, ápice marcadamente comoso. Segmentos del cáliz connatos abaxialmente. Corola glabrescente. Filamentos delgados, bruscamente dilatados en la base de forma deltoidea. Anteras blancas 17. O. densiflora

24. Inflorescencia densa o laxa, ápice redondeado, o escasamente comoso. Segmentos del cáliz libres. Corola pubescente. Filamentos atenuados hacia la base. Anteras moradas o marrones ........... 25

25. Corola 11-16(18) mm. Filamentos abaxiales insertos a (1)1,5-2,5(3) $\mathrm{mm}$ de la base de la corola. Anteras $1-1,4 \mathrm{~mm}$...... 25. O. minor

25. Corola 16-22 mm. Filamentos abaxiales insertos a $(2,5) 3-4(4,5) \mathrm{mm}$ de la base de la corola. Anteras 1,4-1,7 mm

22. O. amethystea

26. Filamentos adaxiales insertos a $(0,5) 1$ $2,5(3,5) \mathrm{mm}$ de la base de la corola, filamentos de base glabra. Estigma bifurcado
14. O. rapum-genistae

26. Filamentos adaxiales insertos a (2)2,5-6 mm de la base de la corola, filamentos de base densamente pelosa, raramente pubérula. Estigma bilobado .... 27

27. Cáliz con segmentos libres, contiguos o superpuestos, o connatos, subenteros o bífidos. Labio superior de la corola escasamente emarginado a veces íntegro, escasamente bilobado ........... 20. O. icterica

27. Cáliz con segmentos libres, separados, bífidos a bisectos, raramente enteros. Labio superior de la corola profundamente emarginado, ampliamente bilobado 28

28. Cáliz con segmentos bífidos a cortamente bipartidos, raramente subenteros, dientes desiguales, lanceolado acuminados. Corola anaranjado rojiza, salmón pálido o color vinoso....

18. O. haenseleri

28. Cáliz con segmentos bisectos a bipartidos, raramente subenteros, dientes subiguales, estrechamente lanceolados de ápice subfiliforme. Corola blanca, generalmente con nerviación color violeta o morado

21. O. crenata

29. Margen de la corola peloso glandular .... 30

29. Margen de la corola glabro o raramente subglabro 33

30. Cáliz con segmentos subenteros, raramente bífidos. Corola ampliamente cilíndrica o campanulada, con pelos glandulares rojo oscuro o morado. Filamentos con pelos rojos o morados 19. O. alba

30. Cáliz con segmentos bífidos, bipartidos o a veces enteros o subenteros. Corola tubulosa, con pelos glandulares de otro color (hialinos o amarillentos). Filamentos con pelos de otro color (hialinos o amarillentos) 31

31. Cáliz 6-10(12) mm, segmentos altamente connatos, contiguos o superpuestos, cortamente bífidos, dientes deltoideos, raramente subulados .......... 13. O. clausonis

31. Cáliz 8-17 mm, segmentos libres, contiguos, o connatos, profundamente bífidos a bipartidos o a veces subenteros, dientes lanceolados con ápice subfiliforme o dientes estrechamente triangulares atenuados ..... 32

32. Planta escasamente pulverulenta. Segmentos del cáliz altamente connatos, a veces contiguos. Filamentos escasamente pelosos en la mitad inferior, glabro o subglabro con escasos pelos glandulares subsésiles en su ápice 23. O. almeriensis 32. Planta pulverulenta. Segmentos del cáliz 
libres, contiguos. Filamentos densa y largamente pelosos en los 2/3(1/2) inferiores, con abundantes pelos glandulares en su ápice 24. O. santolinae

33. Corola generalmente grande de (15)18-28 $\mathrm{mm}$, labio superior profundamente emarginado, ampliamente bilobado, marcadamente patente. Anteras 2-2,5(3) mm 21. O. crenata

33. Corola más pequeña de 11-22 mm, labio superior de emarginado a escasamente emarginado, amplia o brevemente bilobado, patente o porrecto. Anteras 1-2 mm ........ 34

34. Corola 11-16(18) mm. Filamentos abaxiales insertos a (1)1,5-2,5(3) $\mathrm{mm}$ de la base de la corola. Anteras 1-1,4(1,6) mm. 25. O. minor

34. Corola 15-22 mm. Filamentos abaxiales insertos a 2-4(4,5) $\mathrm{mm}$ de la base de la corola. Anteras 1,3-2 mm 35

35. Planta de aspecto pulverulento. Corolas erectas a erecto-patentes. Mitad superior de los filamentos claramente peloso glandular.

24. O. santolinae

35. Planta no pulverulenta. Corolas erectopatentes a patentes. Mitad superior de los filamentos glabros o subglabros 36

36. Segmentos del cáliz libres, separados, raramente contiguos. Espiga de ápice cónico, comoso o subcomoso. Brácteas 14-22(25) $\mathrm{mm}$, mayores que la corola, raramente igualando el labio inferior. Corola frecuentemente inflexa cerca de la base, con dorso uniformemente curvado, a veces +/recto. Filamentos marcadamente inflexos cerca de su mitad ............ 22. O. amethystea

36. Segmentos del cáliz altamente connatos o contiguos. Espiga de ápice redondeado, no comoso. Brácteas 13-16 mm, menores que la corola, a veces igualando el labio inferior. Corola no inflexa cerca de la base, con dorso +/- recto o a veces ligeramente curvado. Filamentos rectos o ligeramente curvados no marcadamente inflexos cerca de su mitad ...

23. O. almeriensis

Subgénero PHELIPANCHE (Pomel)

Tzvelev, in Fl. Evropeiskoi Chasti SSSR, 5: 325 (1981)

Sect. Trionychon Wallr., Schedulae Criticae 1: 314 (1822)

\section{O. ramosa L., Sp. Pl. 633 (1753)}

Phelipanche ramosa (L.) Pomel, Nouv. Mat. Fl. Atl.103 (1874)
Presente en Depresión de Granada, Almería Subdesértica (Pujadas Salvà \& Lora González, 1996: 205; Pujadas Salvà, 2000: 96). Aportamos nuevas citas que amplían o confirman su área de distribución.

GRANADA: Depresión de Granada: Granada, Vega, -1991-, Sáinz, MA 14749. Sierras de Almijara, Guájares y Trevenque: Almuñécar, Torre del Cambrón, 28-IV-1952, H. Roivainen, BC 143760. HUELVA: Andévalo: Huerto de las Palomas, El Granado, 29SPB3352, 18-VI-2001, E. Sánchez Gullón, COA 37543. JAÉN: Sierra Morena: Alrededores de Santa Elena, 1-VIII1933, González Albo, MA 435475. SEVILLA: Vega del Guadalquivir: cercanías de Sevilla, s/f, s/leg, MA 114745.

\section{Categoría UICN: NT}

Observaciones. Es una planta que parasita casi exclusivamente plantas cultivadas (cf. Pujadas Salvà, 2000: 94; 2002: 360), por lo tanto, su presencia en los hábitats que indica Foley (2001b: 39), como matorrales, bordes de camino y zonas ruderales, es muy dudosa. Los apuntes corológicos para la redacción de "Flora iberica" (Foley, 2001b) que hemos podido localizar corresponden a O. nana (Málaga, MGC 31019; Huelva, MGC 8816) o bien a O.mutelii (Almería, MGC 13410; Granada, GDAC 28830; Málaga, MGC 31018)

Valdés (1987: 557) incluye $O$. ramosa, $O$. nana y $O$. mutelii bajo el nombre de $O$. ramosa.

2. O. nana (Reut.) Beck, Biblioth. Bot. 19: 91 (1890)

Phelipanche nana (Reut.) Soják, in Cas. Nar. Muz. (Prague) 140(3-4): 130 (1972)

Presente en Sierra de Aracena, Sierra Morena, Campiña de Huelva y Condado-Aljarafe, Litoral, Valle de Guadalquivir, Subbética Occidental, Sierra del Aljibe, Serranía de Ronda, Axarquía, Sierras de Almijara, Guájares y Trevenque, Depresión de Guadix-Baza y Guadiana Menor, Alpujarras bajas, Contraviesa y Sierra de Gádor, Sierra Nevada y Filabres, Sierras de María, Orce y Estancias, Almería Subdesértica (Pujadas Salvà \& Lora González, 1996: 205-206; Pujadas Salvà, 2000: 97-98). Aportamos nuevas citas que amplían o confirman su área de distribución. (UICN: NT).

ALMERÍA: Almería Subdesértica: 
Rodalquilar, La Polacra, c. Faro, 26-iV-2002, L. Plaza \& Víctor, COA 37545. Mojácar, Playa del Sombrerico, 30SXG0102, $0 \mathrm{~m}, 16-\mathrm{IV}-2005, A$. Pujadas, COA 37546. CÁDIZ: Litoral: Barbate, Pinar de la Breña, 9-III-2002, E. Sánchez Gullón, COA 37547. Chiclana, La Barrosa, parque público, 28-III-2004, D. Rubiales, COA 37548. Chiclana, 10-III-1859, Kalisch, COI-H ${ }^{\circ}$ Willkomm (sub Phelipaea Mutelii F. Schultz, citado en Willkomm, 1870: 629 como Phelypaea ramosa C.A. Mey.). Digues des chemins entre $\mathrm{S}^{\mathrm{a}}$ María et Jerez, 9-IV-1873, R. Fritze, MA 114788. Puerto de Santa María, pinares de Valdelagrana, 16-IV-1973, S. Silvestre, SEV 162961. Barbate, pinares, 27-IV-1978, T. Luque et al., SEV 162911. Valle del Guadalquivir: Bornos, pantano, 15-III1973, J.L. Escudero, SEV 162960. Jerez, Laguna de Medina, 24-IV-1979, B. Cabezudo et al., SEV 162891. Sierra del Aljibe: Los Barrios, base $S^{a}$ del Niño, 27-IV-1983, Ladero et al., SALA 65937. San José del Valle, $S^{\text {a }}$ de las Cabras, Boca de la Foz, 14-V-1979, $200 \mathrm{~m}$, J. Diez \& S. Silvestre, SEV 162952. Serranía de Ronda: Grazalema, Paseo de las encinas y laureles, 28IV-1989, V.E. Martín Osorio, MGC 33145. Entre Villamartín y Prado del Rey, 17-V-1979, P. Candau \& I. Fernández, SEV 162895. Zahara de la Sierra, Garganta de Bocaleones, TF8577, 500 m, 13-V-1983, A. Aparicio et al., SEV 162967. CÓRDOBA: Sierra Morena: Córdoba, Los Villares, 31-X-1995, A. Lora, COA 37549. Córdoba, Baños de Popea, 14-VI-2000, $A$. Pujadas, COA 29504. c. Pantano de la Breña, 17IV-2004, D. Rubiales, COA 3752. El Acebuche, río Guadiato, 21-IV-2004, Ch. Velasco \& Ú. Osuna, COA 37550. Bélmez, 24-III-1978, J. Pastor, SEV 162881. Valle del Guadalquivir: Cerro de Almodóvar, -V-, Ho Amo y Mora, GDA. Posadas, ctra. Posadas a Villaviciosa, c. Arroyo Pajaroncillo, 18-IV-1980, I. Corral \& P. Fernández, SEV 118666. Subbética Occidental: Carcabuey, c. Cortijo Valdecañas, 30SUG8147, 725 m, 18-IV-2001, E. Triano, COA 37553. Rute, Fuente Alta, 10-V-2002, A. Pujadas \& E. Triano, COA 37554. Zuheros, Peña de Miguel Pérez, c. Las Buitreras, 10-IV-2004, COA 37555. GIBRALTAR: Sierra del Aljibe: Gibraltar, 23IV-1841, s/leg, CGE. Campamento, Gibraltar, in littore, IV-1933, Caster, CGE. West side of rock, upper platform of Cable Car, c. $500 \mathrm{ft} ., 24-$ III-
1969, V.H. Heywood et al., SEV 162974. East side, Catalan Bay, 25-III-1969, V.H. Heywood et al., SEV 162973. GRANADA: Sierra Mágina: Deifontes, Sierra de Harana, 30SVG133, 870 m, 8-VI-2000, A. Pujadas \& E. Triano, COA 37544. Alpujarras bajas, Contraviesa y Sierra de Gádor: Motril, Los Tablones, 30 m, 20-V-1975, T. Palomeque, JAEN 630698. Motril, cuneta, 24III-1970, Fernández Casas, MA 412237. HUELVA: Andévalo: Gibraleón, La Majá del Valle, 29SPB7544, 25-IV-2003, E. Sánchez Gullón \& P. Weickert, COA 37556. Sanlúcar de Guadiana, Casa Las Palomas, 29SPB3352, 15V-2001, E. Sánchez, MGC 51212. Campiña de Huelva y Condado-Aljarafe: Pinares de Hinojos, c. Casa del Membrillo, 1-IV-2001, L. Plaza, COA 31081. Hinojos, La Calera, 24-IV-1980, S. Silvestre, SEV 162982. Litoral: Ayamonte, Punta del Moral, 29SPB4616, -IV-2002, E. Sánchez Gullón \& P. Weickert, COA 37559. Umbría sur de Mazagón, Los Hermanillos, Laguna de las Madres, 29SPB8914, 28-IV-2001, E. Sánchez Gullón \& A. Pujadas, COA 37562. Alcornocales de Lepe, 29SPB6128, -2002, E. Sánchez Gullón \& P. Weickert, COA 37650. Punta Umbría, P.N. Enebrales, 29SPB7818, 2-IV-2002, E. Sánchez Gullón \& P. Weickert, COA 37651. Isla Cristina, dunas, 29SPB5219, 25-III-2003, E. Sánchez Gullón \& P. Weickert, COA 37557. Almonte, Turberas de Rivetehilos, 29SQB0812, 5-IV-2003, E. Sánchez Gullón \& P. Weickert, COA 37558. Huelva, Jardín Botánico Dunas del Odiel, 19-IV2006, L. Plaza, COA 37563. JAÉN: Sierra Morena: Andújar a Aldehuela, VH11, 280 m, 27III-1980, C. Fernández López, JAEN 80149 (sub O.mutelii en Molino Olmedo et al., 1995: 50). Andújar, Río de la Cabrera, 30SVH0435, 4-VI1986, E. Cano, JAEN 990416. Marmolejo, 30SUH9910, 300 m, 24-V-1984, E. Cano, JAEN 843801 (sub O.mutelii en Molino Olmedo et al., 1995: 50). Baños de la Encina, presa de Rumblar, 30SVH3024, 400 m, 1-V-1993, S. Peñafiel Trueba, JAEN 930705 (sub O.mutelii en Molino Olmedo et al., 1995: 50). Desfiladero de Despeñaperros, 19-IV-1970, J. Andrés et al., LEB 28813. Valle del Guadalquivir: Torredonjimeno, Arroyo de la Quebrada, VG17, 500 m, 24-IV1977, C. Fernández López, JAEN 77456. Marmolejo, 30SUH9910, 24-V-1984, E. Cano, JAEN 84380. Fuerte del Rey, cerca de Salinas, 
30SVG2095, 350 m, 1-V-1992, A. González Martín, JAEN 920285 (sub O. ramosa en Molino Olmedo et al., 1995: 50). Baeza, Las Escuelas, 30SVG5491, 500 m, 11-V-1996, M.A. Espinosa et al., JAEN 960300. Vilches, Jarabancil, 30SVH5730, 700 m, 25-III-1997, J.L. Hervás Serrano, JAEN 973009. Subbética Occidental: Jaén, Puente de la Sierra, 30SVG3173, 600 m, 17-V-1983, T. Palomeque, JAEN 832434. La Guardia de Jaén, hacia Molino Nuevo, 30SVG4079, $500 \mathrm{~m}$, A. González, 27-IV-1991, JAEN 910520 (sub O. ramosa en Molino Olmedo et al., 1995: 50). Sierra Mágina: Puente del Obispo, Río Bedmar, 320 m, 16-V-1980, C. Fernández López, JAEN 80512 (sub O. mutelii en Molino Olmedo et al., 1995: 50). CazorlaSegura-Las Villas: Quesada a Estación, VG-99, 550 m, C. Fernández López, JAEN 79575 (sub O. mutelii en Molino Olmedo et al., 1995: 50). MÁLAGA: Sierra del Aljibe: Gaucín, ctra. a Jimena, límite provincial, 30S0285709 / 4039406 , 87 m, 13-V-2007, A. Pujadas, COA 37653. Marbella, Puerto Cabopino, UF44, 10 m, 12-III1985, G. Michel, MGC 21238. Júzcar, Jarastepar, 30SUF0460, $1400 \mathrm{~m}, 30-\mathrm{V}-2004$, O. Gavira, MGC 61196. Cartájima, Risos de Cartájima, 30SUF0661, $1350 \mathrm{~m}, 30-\mathrm{V}-2004$, O. Gavira, MGC 61195. Alrededores de Casares, 28-IV1978, T. Luque et al., SEV 162883. Serranía de Ronda: Torcal de Antequera, Los Polvillares, 1V-1987, Jolin \& Carreira, MGC 41223. Ojén, Monte del Juanar, UF3048, 19-V-1990, A. Márquez et al., MGC 31019. Estepona, base $\mathrm{S}^{\mathrm{a}}$ Bermeja, río Padrón, 26-IV-1996, Y. Gil et al., MGC 44301 \& MGC 44302. Ronda, entre Ronda y Atajate, 30-IV-1977, Ladero, SALA 63231. Estación de Gobantes, $\mathrm{S}^{\mathrm{a}}$ de Checa, 370 m, 24IV-1969, E.F. Galiano et al., SEV 162918. Axarquía: Prope urbem Málaga, 1844, Prolongo, COI-H ${ }^{\circ}$ Willkomm (sub Phelipaea caesia Reut.; citado en Willkomm, 1870: 628). Nerja, 30SVF2169, 100 m, 22-IV-2001, M. Sanz Elorza, MA 657243. Depresión de Antequera: Fuente de Piedra, Los Campos, s/f, Ch. Velasco, MGC 47300. La Alameda, Monte de la Mollina, calizas, 510-630 m, 15-IV-1973, S. Talavera \& B. Valdés, SEV 162917. Sierras de Almijara, Guájares y Trevenque: Cómpeta, Venta Panaderos, Balsa de Incendios, VF2078, $750 \mathrm{~m}, 10-\mathrm{IV}-2003, B$. Cabezudo et al., MGC 59647. SEVILLA: Sierra
Morena: Entre Gerena y El Esparragal, 29-IV1982, A. Aparicio et al., SEV 162968. Vega del Guadalquivir: Sevilla, Barrio Santa Cruz, 1-IV2004, J. Salcedo, COA 37565. Valle del Guadalquivir: Osuna, El Cantalejo, 12-IV-2001, L. Plaza, COA 37564. Estepa, Pico Becerro, 15IV-1973, S. Talavera \& B. Valdés, SEV 162916. Utrera, Pantano de Torre del Águila, 4-IV-1977, E.F. Galiano et al., SEV 162890. Carmona, Ermita de Santa $M^{\text {a }}$ de Gracia, 24-IV-1979, $P$. Candau et al., SEV 162889. Morón de la Frontera, Pico Esparteros, 21-V-1980, J. Díez et al., SEV 162985.

Observaciones. No citada expresamente por Valdés (1987) en Andalucía Occidental.

3. O. olbiensis (Cosson) Nyman, Syll. Fl. Eur. : 133 (1854)

Phelipaea olbiensis Coss., Not. Pl. Crit.: 8 (1849) [basión]

Ph. ramosa subsp. mutelii var. olbiensis (Coss.) Rouy, Fl. France 11: 160 (1909)

Ph. ramosa subsp. olbiensis (Coss.) Bonnier, Fl. Ill. France 8(78): 91 (1926)

Citada recientemente por Pujadas Salvà (2006: 247) en la provincia de Almería, Almería Subdesértica, como nueva cita para la Península Ibérica. Aportamos una nueva recolección que confirma su presencia.

ALMERÍA: Almería Subdesértica: Roquetas, Punta del Sabinar, c. Faro, $36^{\circ} 41,155^{\text {' }}$ N, $2^{\circ} 41,994^{\prime} \mathrm{W}, 0$ m, 17-III-2007, A. Pujadas, A. Ivorra \& P. Soria, COA 37656.

Categoría UICN: CR [B2ac(iv)]

Observaciones. No citada por Foley (2001b). Schneeweiss et al., (2004, suplementary data: 10) identifican la recolección WU 25267 como O. rosmarina Beck (Pujadas Salvà, 2006: 247).

4. O. mariana A. Pujadas, Acta Bot. Malacitana 32: 269-274.

Orobanche rosmarina sensu auct. hisp., p. p., non Beck in Ginzberger, Oesterr. Bot. Z. 70(9/ 12): 243.1921.

Descrita recientemente por Pujadas Salvà (2007b: 269-274), citada en las provincias de Córdoba y Sevilla, en Sierra Morena.

Observaciones. Citada erróneamente como 
O. rosmarina Beck en Pujadas et al. (2005). Categoría UICN: VU [B1ac(iv); D1+2]

5. O. mutelii F.W. Schultz in Mutel, Fl. Franç. 2: 353 (1835)

- Planta pubescente glandular. Tallo escasamente engrosado de $(1,5) 2-4 \mathrm{~mm}$ de diámetro en su mitad. Inflorescencia laxa o densa. Brácteas 7-9 mm. Bractéolas 6-7 mm. Corola pubescente glandular. Anteras 1,3-1,6 $\mathrm{mm}$ a. subsp. mutelii

- Planta densamente pubescente a cortamente aracnoidea glandular, frecuentemente de aspecto pulverulento. Tallo robusto de 5-9 $\mathrm{mm}$ de diámetro en su mitad. Inflorescencia densa. Brácteas 10-13 mm. Bractéolas 7-10 $\mathrm{mm}$. Corola densamente viloso glandulosa. Anteras 1,5-2 mm $\boldsymbol{\beta}$. subsp. georgii-reuteri

\section{$\alpha$. subsp. mutelii}

Phelipanche mutelii (F.W. Schultz) Pomel, Nouv. Mat. Fl. Atl. 106 (1874)

Presente en Litoral, Valle de Guadalquivir, Subbética Occidental, Sierra de Mágina, CazorlaSegura-Las Villas, Sierra del Aljibe, Serranía de Ronda, Axarquía, Sierras de Almijara, Guájares y Trevenque, Sierra Nevada y Filabres, Sierras de María, Orce y Estancias, Altiplanos Manchegos y Almería Subbética, Almería Subdesértica (Pujadas Salvà \& Lora González, 1996: 206; Pujadas Salvà, 2000: 99-100). Aportamos nuevas citas que amplían o confirman su área de distribución. (UICN: NT).

ALMERÍA: Almería Subdesértica: El Alquián, 1-IV-1986, M. Cueto, ALME 17791 \& ALME 17793. Las Negras, Cala del Cuervo, 19IV-2002, A. Pujadas, COA 37473. Playa del Pozo del Esparto, 30SXG1636, 0 m, 14-IV-2005, A. Pujadas, COA 37537. Mojácar, Playa del Sombrerico, 30SXG0102, 0 m, A. Pujadas, COA 37538. Carboneras, 27-VI-1976, T. Palomeque, JAEN 630661 \& JAEN 630663. CÁDIZ: Litoral: Inter Pto. Santa María et Jerez, 9-IV-1873, $R$. Fritze, PRC. Serranía de Ronda: Grazalema, hacia Zahara, $1200 \mathrm{~m}, 17-\mathrm{V}-1987$, G. Mateo et al., VAL 146141. GIBRALTAR: Sierra del Aljibe: Gibraltar, sandy banks, 24-III-1841, C.M. Lemann, CGE. GRANADA: Subbética Occidental: Montillana, cortijo de Las Juntas, 30SVG4147, 1120 m, 23-V-1970, C. Fernández López, JAEN. Sierra Mágina: Huélago, 16-VII-
1971, Fernández Casas, MA 412302. CazorlaSegura-Las Villas: Cazorla, Cima de la Cabrilla, 30SWG1694, 2030 m, 12-VII-1985, C. Fernández \& J. Cobos, JAEN 851578 \& JAEN 851611. S a de la Sagra, WG 3699, 1850-2300 m, 24-VI-1988, B. Valdés et al., SEV s/n (en Valdés \& Talavera, 1991: 205). Axarquía: Almuñécar, La Herradura, Cerrogordo, 30 m, 5-IV-1976, T. Palomeque, JAEN 630692 \& JAEN 630696. Sierras de Almijara, Guájares y Trevenque: Cogollos Vega, Las Escurridizas, 30SVG5028, 1000 m, 17-IV-1988, A.M. Negrillo \& L. Cano, GDA 22362. Almuñécar, La Herradura, Punta de la Mona, 20-IV-1988, E. Arrojo Agudo, GDAC 33296. Alpujarras bajas, Contraviesa y Sierra de Gádor: Granada a Motril, Km 48.1, 20-V-1975, T. Palomeque, JAEN 630633. HUELVA: Sierra de Aracena: Aracena, 1-IV-1984, F. Hinojosa, SEV 162970. Campiña de Huelva y CondadoAljarafe: Rociana del Condado, 4-V-1977, J. Andrés, LEB 60865. JAÉN: Subbética Occidental: Jaén, Fuente de la Peña, VG27, 700 m, 29-V-1975, C. Fernández López, JAEN 75509. Jaén, Puente de la Sierra, 30SVG3173, 600 m, 17-V-1983, T. Palomeque, JAEN 832434. Valdepeñas de Jaén, Los Rosales, 30SVG3052, 1200 m, 20-VI-2002, C. Fernández, JAEN 620357 (bis). Sierra de Mágina: Bélmez de la Moraleda, Sierra de la Cruz, roca del Neblí, 15VII-1971, J. Fernández Casas, MA 412348. Cazorla-Segura-Las Villas: Cazorla, Cima de la Cabrilla, 30SWG1694, 2030 m, calizas, 12-VII1985, C. Fernández \& J. Cobos, JAEN 851578 y 851611. Sierra Seca, El Chaparral, WH2800, 1800 m, 29-VI-1988, B. Valdés et al., SEV s/n (en Valdés \& Talavera, 1991: 205). MÁLAGA: Sierra del Aljibe: Marbella, sandy seashore, IV1931, Goulay et al., GCE. Marbella, uncultured rocky ground, IV-1936, P.W. Richards, CGE. Benarrabá, Los Pepes, 30STF9744, 24-III-2001, O. Gavira, MGC 49225. Serranía de Ronda: Sierra Blanca, Ventorro la Chata, 7-V-1982, Merino \& Guerra, MGC 11781 (junto a otros especímenes de $O$. nana). Alhaurín de la Torre, $\mathrm{S}^{\mathrm{a}}$ de Mijas, Los Caracolillos, 12-IV-1984, B. Cabezudo et al., MGC 16532. Alhaurín el Grande, $\mathrm{S}^{\mathrm{a}}$ Mijas, 12-IV-1984, B. Cabezudo et al., MGC 16533. Axarquía: La Araña, fábrica de cemento, 30SUF8264, 50 m, 28-III-2003, O. Gavira, MGC 58350. Depresión de Antequera: La Alameda, 
Monte de la Mollina, 510-630 m, 15-IV-1973, S. Talavera \& B. Valdés, SEV 162917 (junto a otros especímenes de $O$. nana). Humilladero, $\mathrm{S}^{\mathrm{a}}$ del Humilladero, UG 50, 600 m, 24-III-1988, J.R. Boyero, MGC 64169. SEVILLA: Valle del Guadalquivir: Alcalá de Guadaira, 8-IV-1973, B. Cabezudo, SEV 162900. Mairena del Alcor, 26IV-1975, E. Ruiz de Clavijo, SEV 162921. Carmona, Ermita de Santa $\mathrm{M}^{\mathrm{a}}$ de Gracia, 24-IV1979, P. Candau et al., SEV 162889. Entre Herrera y Écija, Las Cañuelas, T. Luque et al., SEV 162986.

Observaciones. No citada expresamente por Valdés (1987) en Andalucía Occidental.

及. subsp. georgii-reuteri (L. Carlón, G. Gómez Casares, M. Laínz, G. Moreno Moral, Ó. Sánchez Pedraja \& G.M. Schneeweiss) A. Pujadas, Acta Bot. Malacitana 32: 265-267. Orobanche georgii-reuteri (L. Carlón, G. Gómez Casares, M. Laínz, G. Moreno Moral, Ó. Sánchez Pedraja \& G.M. Schneeweiss) A. Pujadas, Lagascalia 26: 246 (2006)

O. mutelii var. spissa auct. hisp., non Beck, Mon. Orob. : 96 (1890)

Phelipanche georgii-reuteri L. Carlón, G. Gómez Casares, M. Laínz, G. Moreno Moral, Ó. Sánchez Pedraja \& G.M. Schneeweiss, Documentos Jard. Bot. Atlántico (Gijón) 3: 1718 (2005) [basión.]

Citada recientemente en Cazorla-SeguraLas Villas, Depresión de Guadix-Baza y Guadiana Menor y Sierras de María, Orce y Estancias (Pujadas Salvà, 2006: 246). Añadimos la siguiente referencia:

GRANADA: Depresión de Guadix-Baza y Guadiana Menor: Benamaurel, alrededores, yesos y margas, 30SWG2662, $700 \mathrm{~m}, 22-\mathrm{V}-2004, C$. Fernández, M. Carazo \& V. Gómez, JAEN 640484.

\section{Categoría UICN: DD}

Observaciones. Muy próximo a $O$. muteli subsp. mutelii y en algunos casos es difícil su separación por lo que se requieren estudios más profundos para definir las relaciones entre ambos taxones.

6. O. lavandulacea Rchb., Iconogr. Bot. Pl. Crit. 7: 48 (1831)

Phelipanche lavandulacea (Rchb.) Pomel,
Nouv. Mat. Fl. Atl. 106 (1874)

Presente en Sierra Morena, Subbética Occidental (Pujadas Salvà, 2000: 100). Aportamos nuevas citas que amplían su área de distribución.

CÁDIZ: Serranía de Ronda: $S^{a}$ del Pinar, entre Grazalema y Zahara de la Sierra, 5-V-1983, S. Talavera \& B. Valdés, SEV 162941. JAÉN: Subbética Occidental: Alcalá la Real, Arroyo Juan Castillo, 30SVG2248, 8-V-1993, M. Espinosa Gento \& $M^{a}$ J. Fernández, JAEN 931495 (sub $O$. ramosa en Molino Olmedo et al. 1995: 50).

Categoría UICN: VU [B1ac(iv); D1+2]

Observaciones. No citada por Valdés (1987) en Andalucía Occidental.

7. O. schultzii Mutel, Fl. Franç. 2: 429 (1835) Phelipanche schultzii (Mutel) Pomel, Nouv. Mat. Fl. Atl. 107 (1874)

Phelypaea schultzii (Mutel) Walp., Repert. Bot. Syst. 3: 463 (1844)

Presente en Andévalo (Sánchez Gullón et al., 2005: 255) y en Litoral, Subbética Occidental, Sierra Mágina, Serranía de Ronda, Axarquía, Sierras de María, Orce y Estancias (Pujadas Salvà \& Lora González, 1996: 208; Pujadas Salvà, 2000: 102-103). Aportamos nuevas citas que amplían o confirman su área de distribución.

CÁDIZ: Litoral: Chiclana, La Barrosa, parque público, 28-III-2004, D. Rubiales, COA 37541. CÓRDOBA: Sierra Morena: Espiel, Sierra de Espiel, 30S0321601 / 4226378, 612 m, 30-IV-2007, E. Reyes Sepúlveda \& A. Pujadas, COA 37642. Los Villares, Parque Periurbano, 341893 / 4206080, 600 m, 28-V-2007, E. Reyes Sepúlveda, COA 37661. Subbética Occidental: Rute, base de la Sierra Alta, pinar de Rute, 10-V2002, E. Triano \& A. Pujadas, COA 37479. GRANADA: Sierra de Mágina: Torre Cardela a Moreda, 30SVG6949, 17-VI-2000, E. Morales Ramos \& C. Fernández López, JAEN 701075. Campotéjar a Cortijo Molina, 30SVG4547, 1100 m, 20-VI-2000, C. Fernández López, JAEN 700415. HUELVA: Andévalo: Sanlúcar del Guadiana, Casa de la Madrina, 29SPB3650, 15V-2001, E. Sánchez Gullón, COA 31015. Ayamonte, talud margen Guadiana, 29SPB4121, 21-III-2002, E. Sánchez Gullón \& A. Pujadas, COA 37542. Sanlúcar del Guadiana, La Rochona, 29SPB3550, 15-V-2001, E. Sánchez Gullón, 
MGC 51211. Ayamonte, alrededores, márgenes del Guadiana, 18-V-1979, S. Silvestre et al., SEV 162923. JAÉN: Subbética Occidental: Valdepeñas de Jaén, Los Rosales, 30SVG3052, 1200 m, 20-VI-2002，C. Fernández, JAEN 620357. MÁLAGA: Axarquía: Pizarra, Hacho de Pizarra, SW, 200-250 m, 20-IV-2006, A. Pérez Latorre et al., MGC 64776. Alora, 25-VI-1873, Winkler, PRC.

Categoría UICN: NT

Observaciones. No citada por Valdés (1987) en Andalucía Occidental.

8. O. purpurea Jacq., Enum. Stirp. Vindob.: 108 (1762)

Phelipanche purpurea (Jacq.) Soják, in Cas. Nar. Muz. (Prague). 140(3-4): 130 (1972)

Presente en Sierra Mágina (Pujadas Salvà, 2000: 105), única localidad conocida en Andalucía. $\mathrm{C} 2 \mathrm{~b}]$

Categoría UICN: CR [B1ab(v) + 2ac(iv);

Observaciones. Citada erróneamente por Foley (2001b: 46) para Almería, referencia que recogen Cabezudo et al. (2005: 99). El pliego testigo que ha dado lugar a esta cita, "Base de la $\mathrm{S}^{\mathrm{a}}$ Cabrera, playa del Castillejo de Macenas, entre albaidal, 30SXG0204, 5-IV-1990, J. Peñas \& $M^{a} . J$. Salinas, 5-IV-1990, GDAC 33375", corresponde a ejemplares de $O$. mutelii. Además el lugar de recolección difiere notablemente del hábitat de $O$. purpurea de clima más templado y húmedo.

La cita de Willkomm (1870: 628), sub Phelipaea caerulea C.A. Meyer, de "La Albaida, pr. Cordova, AMOR, teste LGE" probablemente corresponda a $O$. nana (Reut.) Beck.

Las citas de Valdés \& Talavera (1991: 204) para las provincias de Granada y Jaén correponden a $O$. arenaria Borkh.

\section{O. arenaria Borkh., Neues Mag. Bot.: 6 (1794)}

Phelipanche arenaria (Borkh.) Pomel, Nouv. Mat. Fl. Atl. 103 (1874)

Presente en Sierra de Mágina, CazorlaSegura-Las Villas, Depresión de Granada, Trevenque, Alpujarras bajas, Contraviesa y Sierra de Gádor, Sierra Nevada y Filabres, Sierra de Baza, Sierra de María, Orce y Estancias, Altiplanos Manchegos y Almería Subbética, Almería Subdesértica (Pujadas Salvà \& Lora
González, 1996: 208, sub O. laevis L.; Pujadas Salvà, 2000: 103-104). Aportamos nuevas citas que amplían o confirman su área de distribución.

ALMERÍA: Altiplanos Manchegos y Almería Subbética: Vélez Blanco, km 1 de Vélez Blanco a Vélez Rubio, 30S0579738 / 4170805, $1070 \mathrm{~m} \mathrm{~A}$. Pujadas \& A. Ivorra, COA. GRANADA: Cazorla-Segura-Las Villas: Huéscar, claro de pinar, 1200 m, 2-VI-1996, D. Guijarro, COA 9480. Castril, Fátima, La Azadila, 30SWG2488, 1260 m, 19-VI-2004, Fernández, JAEN 640979. Huéscar, San Clemente, orilla pantano, 30SWG3091, $1080 \mathrm{~m}, 25-\mathrm{VI}-2004, C$. Fernández et al., JAEN 641110. Huéscar, $\mathrm{S}^{\mathrm{a}}$ de Moncayo, 30SWG3698, 1540 m, 30-VI-2004, C. Fernández et al., JAEN 641158. Sa de la Sagra, 30SWH3703, 1500 m, 19-VII-1977, E. ValdésBermejo \& S. Castroviejo, MA 435505. Sierras de Almijara, Guájares y Trevenque: Sierra de las Almijaras supra pagum Gujar Alto 2000', 17-VI1845, Willkomm, COI-H ${ }^{\circ}$ Willkomm (sub Phelipaea arenaria Walp.; citado en Willkomm, 1870: 628). Alpujarras bajas, Contraviesa y Sierra de Gádor: La Alpujarra, V-1902, C. Pau, MA 435789. Sierra Nevada y Filabres: Lugros, Dehesa del Camarate, 19-VI-2003, A. Pujadas \& L. Plaza, COA 37480. JAEN. Cazorla-SeguraLas Villas: Santiago de la Espada, Pte. Río Zumeta, 30SWH3818, 1280 m, 24-VII-1996, A. Camacho \& A. Fernández Ocaña, JAEN 960991. MÁLAGA: Sierras de Almijara, Guájares y Trevenque: Canillas de Albaida, laderas del Río Cájula, 30SVF1178, $500 \mathrm{~m}, 12-\mathrm{V}-2005, G$. Caballero \& F.C. Soriguer, MGC 63898.

Categoría UICN: NT

Observaciones. El pliego de "Sevilla, s/f, J. Rodríguez, MA 114785" (Pujadas Salvà 2000: 104) aunque corresponde a $O$. arenaria, es una referencia dudosa o un error de etiqueta ya que la planta no se ha vuelto a encontrar en toda Andalucía Occidental.

Subgénero BOULARDIA (F.W. Schultz) A. Pujadas, Acta Bot. Malacitana 32 : 265-267. Género Boulardia F.W. Schultz, Arch. Fl. France Allem. 103 (1847) [basión.]

10. O. latisquama (F.W. Schultz) Batt. in Batt. \& Trab., Fl. Algérie 1: 659 (1890) Presente en Alpujarras bajas, Contraviesa y 
Sierra de Gádor, Sierra Nevada y Filabres, Sierras de María, Orce y Estancias, Almería Subdesértica (Pujadas Salvà \& Lora González, 1996). Aportamos nuevas citas que amplían o confirman su área de distribución. (UICN: NT).

ALMERÍA: Alpujarras bajas, Contraviesa y Sierra de Gádor: Sierra de Gádor, Barranco del Caballar, 30SWF4578, 170 m, F.J. Pérez-García, HUAL 6911. Sierras de María, Orce y Estancias: Lúcar, Montroy, 30SWG5239, 11-V-1996, F. Navarro Reyes, GDAC 43037. Almería Subdesértica: Carboneras-Níjar, Km 10, 30SWF9092, 13-III-1994, A. Pujadas \& P. Poyato, COA 17441. Rodalquilar, La Polacra, 26IV-2002, L. Plaza, COA 37507. Macael, $500 \mathrm{~m}$, 20-V-1976, B. Cabezudo et al., SEV 162931. CÁDIZ: Valle del Guadalquivir. Morón de la Frontera a Coripe, Km 5, 30-III-1997, J. Pusch, COA 23588. Cerros de Bornos, 28-I-1993, J. Herrera \& S. Talavera, SEV 136802. CÓRDOBA: Sierra Morena: Obejo, río Guadalbarbo, c. Cortijo del Cura, 30SUH4117, 290 m, 18-V-2001, A. Pujadas \& D. Rubiales, COA 37501. Córdoba, Arroyo Bejarano, 21-IV2004, Ch. Velasco \& Ú. Osuna, COA 38417. Córdoba, Los Villares, 30SUH4208, 550 m, 30IV-2007, E. Reyes Sepúlveda \& A. Pujadas, COA 37647. Subbética Occidental: Base La Tiñosa, 15V-1991, A. Lora \& A. Pujadas, COA 17434. Carcabuey, Sierra Alcaide, 30SUG8648, $700 \mathrm{~m}$, 14-XI-2001, E. Triano \& A. Pujadas, COA 37508. Lucena, Cerro de la Galeota, 18-IV-1980, Díaz \& Muñoz, COFC 5957 \& SEV 162977. Priego de Córdoba, Sierra de Pollos, UG84, 2-V-1980, J. Muñoz, COFC 5956. GRANADA: Sierra Mágina: Iznalloz, próximo al Cortijo de la Inquisición, 30SVG4741, 1000 m, 20-V-1989, F.J. Garcia \& L. Gutiérrez, GDAC 37115. Cazorla-Segura-Las Villas: Castril, Fátima, La Azadilla, 30SWG2488, 1260 m, 19-VI-2004, Fernández et al., JAEN 640967. Depresión de Granada: Dílar, 10-V-1975, T. Palomeque, JAEN 630670 \& JAEN 630675. Sierras de Almijara, Guájares y Trevenque: Alhama, 16-VI-1991, $A$. Pujadas \& P. Poyato, COA 17438. Playa de Cantarriján, 10 m, 22-IV-1995, A. Pujadas, COA 17437. Cortijo Corvales, c. La Zubia, 1000 m, 3V-2002, C. Burgarella \& L. Plaza, COA 37506. Sierra de Alfacar, 7-V-1977, F. Valle, GDAC 9310. Almuñécar, P.N. Sierra Tejeda, Almijara y
Alhama, Cerro de los Gigantes, VF3173, $750 \mathrm{~m}$, 16-V-2003, B. Cabezudo et al., MGC 59650. Depresión de Guadix-Baza y Guadiana Menor: Cerro de Jabalcón, près Baza, 30-V-1851, E. Bourgeau, COI-H ${ }^{\circ}$ Willkomm (sub Ceratocalyx macrolepis Coss.; citado en Willkomm, 1870: 631). Los Balcones, Dehesa de los Cuartos, 30SWG0347, 1030 m, 24-IV-1998, F.B. Navarro Reyes, GDA 47711. Alpujarras bajas, Contraviesa y Sierra de Gádor: Azud de Vélez, cerca de Vélez de Benaudalla, 25-III-1988, C. Morales et al., GDAC 32919. Almuñécar, La Herradura, Cerrogordo, 30 m, 5-iV-1976, T. Palomeque, JAEN 630690 \& JAEN 630693. Sierra Nevada y Filabres: Sierra Nevada. Pista Barranco de los Alisos, a $2 \mathrm{Km}$ de Nigüelas, 22-VII-1997, $P$. Prados et al., COA 23559. Sierra de Baza: Morrón Alto, 30SWG1444, 1400 m, 11-VI-2002, F.B. Navarro \& C. Morales, GDA 45991. JAÉN: Citada por Molino Olmedo et al., (1995), añadimos las siguientes localidades. Sierra Morena: Sierra Quintana, río Valmaya, 400220 / 4248161, 27-IV-2004, L. Plaza \& Ch. Velasco, COA 37505. Andújar, Rosalejillo, 9-V-1979, T. Palomeque, JAEN 630688. Andújar, Cerro Madroño, 30SVH0822, $440 \mathrm{~m}, 11-\mathrm{V}-1993, T$. Carrera \& P. Díaz, MA 652010. Valle del Guadalquivir: Vilches, Cerro del Manzano, 30SVH5628, 500 m, 19-III-1997, J.L. Hervás Serrano, JAEN 973005. Subbética Occidental: Subida a La Pandera, 1600 m, 11-VI-1991, $A$. Pujadas \& A. Lora, COA 17436. Fuensanta de Martos, VG16, 600 m, 12-VI-1976, C. Fernández López, JAEN 76182. Sierra Mágina: La Silleta, 1200 m, 10-VI-1925, Cuatrecasas, BC 46571. Barranco del Ronco in Sierra de la Cruz, 1200 m, 12-VI-1925, Cuatrecasas, BC 46570. Cerro Gordo, 1200 m, 17-VI-1925, Cuatrecasas, BC 46572. Sa Mágina, Barranco de Tosquilla, 1-VIII1996, JLV \& AJ, COA 37504. Huelma, Camino del Gargantón, 18-V-2003, COA 37503. Cabra de Santo Cristo, 30S0476556 / 4181113, 28-IV2006, A. Pujadas \& L. Plaza, COA 37533. Cazorla-Segura-Las Villas: Sierra de Cazorla, c. pico la Viñuela, 30SWG0599, 1200m, A. Lora, COA 22098. Sierra de Cazorla, Mirador del puerto de las Palomas, 6-V-1992, A. Pujadas \& A. Lora, COA 22103. Cazorla, Jardín Botánico, El Hornico, 15-V-1997, P. Luque, COA 23753. Sierra de Cazorla, El Cantalar, 17-V-2002, L. 
Plaza et al., COA 37502. Orcera, Arroyo Fuente de la Zarza, 30SWH3445, $1000 \mathrm{~m}, 1-\mathrm{V}-1997$, J.C. Rodríguez Zorrilla, JAEN 620167. Cortijos Nuevos, El Yelmo, 1200 m, 30-VI-1988, A. Aparicio et al., SEV 162908. MÁLAGA: Serranía de Ronda: Antequera, Sierra Llana, 30SUF4290, 21-III-1997, L. Plaza et al., COA 23364. Marbella, Sierra Blanca, La Alpujata, 3V-2003, P. Blanco, COA 37509. Prope pagum Fuengirola, 28-IV-1845, Willkomm, COI-H ${ }^{\circ}$ Willkomm (sub Orobanche cruenta Boiss.; citado en Willkomm, 1870: 631, como Ceratocalyx macrolepis Coss.). Ronda, Tajo del Abanico, UF0465, 680 m, 16-IV-1988, D. Montilla, MGC

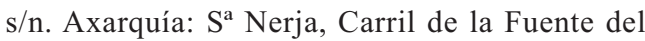
Esparto, 22-IV-1983, M. Trigo, MGC 10955. Cerro San Antón, 10-IV-1987, E. Ferrer et al., MGC 22241. Depresión de Antequera: Humilladero, Sa de Humilladero, UG 50, 24-III1988, J.R. Boyero, MGC 64169. Sierras de Almijara, Guájares y Trevenque: Frigiliana, P.N. Sierra Tejeda, Almijara y Alhama, balsa de los incendios al Puerto de los Umbrales, VF2177, 900 m, 23-V-2003, B. Cabezudo et al., MGC 59646. Nerja, P.N. Sierra Tejeda, Almijara y Alhama, subida al Tajo del Almendrón desde Fuente del Esparto, 30SVF2474, 900-1100 m, 6-V-2004, B. Cabezudo et al., MGC 59654. SEVILLA: Valle del Guadalquivir: Coripe, Río Guadalporcum, 12III-1977, M.J. Gallego et al., COFC 4471 \& SEV 31881. Morón, Sierra de Esparteros, 15-IV-1977, E. Ruiz de Clavijo, MAF 102143.

Subgénero OROBANCHE L., Sp. P1. 632 (1753) Sect. Osproleon Wallr., Schedulae Criticae 1: 307 (1822)

11. O. cernua L. in Loefl., Iter. Hisp. 152 (1758)

Presente en Sierra Mágina, Sierra de Almijara, Guájares y Trevenque, Alpujarras bajas, Contraviesa y Sierra de Gádor, Sierra Nevada y Filabres, Sierra de María, Orce y Estancias, Almería Subdesértica (Pujadas Salvà \& Lora González, 1996: 209-210; Pujadas Salvà \& Velasco, 2000: 515-516). Aportamos nuevas citas que amplían o confirman su área de distribución.

ALMERÍA: Alpujarras bajas, Contraviesa y Sierra de Gádor: Fuente Cufría, 17-V-1974, $R$. Sagredo, $\mathrm{H}^{\circ}$ La Salle Almería. Barranco del Caballar, 30SWF4578, 150 m, 2-IV-1999, F.J.
Pérez-García, HUAL 6912. Sierra Nevada y Filabres: Benizalón, Finca de los Pajares, 30SWG6617, 800 m, 15-II-1988, Morales \& A.B. Robles, GDA 49016. Castro de Filabres, Sierra de los Filabres, Rambla de Castro, 30SWG4914, 850 m, 19-IV-1993, J. Peñas, HUAL 6894. Altiplanos Manchegos y Almería Subbética: Vélez Blanco, de El Gabar a Vélez Blanco, 30S0578448 / 4174018, 1030 m, 26-IV-2007, A. Pujadas \& A. Ivorra, COA 37664. Almería Subdesértica: Sierra Alhamilla, 23-VII-1996, L. Plaza et al., COA 37531. Sierra Almagrera, 30SXG1129, 162 m, 14-IV-2005, A. Pujadas, COA 37529. Mojácar, Playa del Sombrerico, 30SXG0102, 0 m, 16-IV-2005, A. Pujadas, COA 37530. Sorbas, Río Aguas, 30SW68105, $400 \mathrm{~m}$, 21-III-1998, F.J. Pérez-García, HUAL 6913. Gérgal, Desierto de Tabernas, c. Colorada, 30SWG4205, 3-V-2002, J. Peñas, HUAL 7299. CÓRDOBA: Subbética Occidental: Priego de Córdoba, Solvito, 30S0403534 / 4150830, 480 m, 1-V-2007, A. Pujadas \& E. Triano, COA 37643. GRANADA: Subbética Occidental: Sierra de Parapanda, 30SVG1831, 1400 m, 6-VI-2000, $A$. Pujadas \& E. Triano, COA 29900. CazorlaSegura-Las Villas : Puebla de D. Fadrique, cerro del Po..[ilegible], calizas, 30SWG5299, $1100 \mathrm{~m}$, 14-VI-2005, C. Fernández, JAEN 650675. Sierras de Almijara, Guájares y Trevenque: Salobreña, 30-III-2003, D. Rubiales, COA 33960. Depresión de Guadix-Baza y Guadiana Menor: Cúllar-Baza, El Margen, 30SWG3766, 80 m, 8VI-2000, A. Pujadas, COA 29902. Coteaux arides près Baza, 28-V-1851, E. Bourgeau, COI-H ${ }^{\circ}$ Willkomm (citado en Willkomm, 1870: 627). Prope Baza, 1853, Rossmaessler, COI-H ${ }^{\circ}$ Willkomm (citado en Willkomm, 1870: 627). Margen de Arriba, 30SWG3766, 860 m, 8-VI2000, P. Montserrat \& L. Villar, JACA 206800. Gorafe, 30SWG9552, $880 \mathrm{~m}, 22-\mathrm{IV}-2001, C$. Fernández López \& I. García Abasolo, JAEN 610206. Bacor hacia Zújar, 30SWG1055, $740 \mathrm{~m}$, 5-V-2004, C. Fernández et al., JAEN 640252. Alpujarras bajas, Contraviesa y Sierra de Gádor: Motril, Los Tablones, 30 m, 20-V-1975, T. Palomeque, JAEN 630697. HUELVA: Litoral: Espigón de Punta Umbría, 29SPB8216, 20-III2000, E. Sánchez Gullón, COA 29589. Laguna de Palos, 29SPB8716, 14-IV-2000, E. Sánchez Gullón, COA 29588. JAÉN: Sierra Mágina: 
Cerro Cárceles, vertiente Norte, 1600-1900 m, 2VIII-1996, J.L. Vivero \& A.J., COA 37532. Depresión de Guadix-Baza y Guadiana Menor: Larva a San Pedro, 540 m, 11-VI-1975, T. Palomeque, JAEN 630662. Hinojares, 30SWG0073, 700 m, 4-V-1996, L. Morillas \& C. Fernández, JAEN 962074. SEVILLA: Valle del Guadalquivir: Carmona, 30STG6250, 204 m, 6IV-2002, Sánchez Pedraja \& M. Tapia, $\mathrm{H}^{\circ}$ Sánchez Pedraja 10388.

Categoría UICN: LC

Observaciones. No citada por Valdés (1987) en Andalucía Occidental.

12. O. cumana Wallr., Orob. Gen. Diask. 58 (1825)

Presente en Campiña de Huelva y CondadoAljarafe, Vega del Guadalquivir, Valle del Guadalquivir, Depresión de Antequera, Depresión de Granada (Pujadas Salvà \& Velasco, 2000: 517519). Aportamos nuevas citas que confirman su área de distribución.

CÓRDOBA: Valle del Guadalquivir : De Espejo a Montemayor, c. Ermita Dos Hermanas, 30SUG5768, A. Pujadas \& J. Fuertes, COA 33962. HUELVA: Campiña de Huelva y Condado-Aljarafe: Escacena, 23-VI-2005, E. Sánchez-Gullón, COA 33961.

Categoría UICN: LC

Observaciones. No citada por Valdés (1987) en Andalucía Occidental. Foley (2001b: 49) la incluye en $O$. cernua.

13. O. clausonis Pomel, Bull. Soc. Sci. Phys. Algérie 11: 107 (1874)

Presente en Sierra de Aracena, Subbética Occidental, Sierra Mágina, Serranía de Ronda, Axarquía (Pujadas Salvà \& Lora González, 1997: 478). Aportamos nuevas citas que amplían o confirman su área de distribución conocida.

CÁDIZ: Serranía de Ronda: Algodonales, Sierra de Líjar, 31-V-1979, A. Aparicio et al., SEV 162964. Grazalema, El Pinsapar, 19-VI1990, M. Arista \& P.L. Ortiz, SEV 136900. Grazalema, Sierra del Pinar, 9-VII-1991, M. Arista, SEV 135002. GRANADA: Sierra Nevada y Filabres: Lugros, Dehesa del Camarate, 30SVG7116, 1450 m, 19-VI-2003, A. Pujadas \& L. Plaza, COA 33959. JAÉN: Subbética Occidental: Castillo de Locubín, La Gitana,
30SVG1756, 820 m, 22-V-1993, J.M. Espinosa Gento, JAEN 932714 (sub O. foetida en Molino Olmedo et al., 1995: 48). Cazorla-Segura-Las Villas: Cortijos Nuevos, El Yelmo, 1200 m, 30VI-1988, Aparicio et al., SEV 162910. MÁLAGA: Sierra del Aljibe: Geralguacil, Sierra Bermeja, Puerto de Peñas Blancas, 4-VI-1991, P. Navas, MGC 44098. Benarrabá, Arroyo de Las Cobatillas, 30STF9646, 300-400 m, 29-V-2004, O. Gavira, MGC 61194. Genalguacil, Monte del Duque, 30STF9744, 7-V-2006, O. Gavira, MGC 64771. Serranía de Ronda: Villanueva del Rosario, Sierra del Jobo, pico del Jobo, 30SUF8293, 1300 m, 11-VI-1996, $Y$. Gil \& $A$. Pérez-Latorre, MGC 42070. Yunquera, P.N. Sierra de las Nieves, El Camón, cabecera del Arroyo de los Zarzalones, 30SUF2464, 11001200 m, 3-IV-1997, Y. Gil et al., MGC 46060. Axarquía: Málaga, Montes de Málaga, entre Venta de Galmey y ctra. de Olías, 30SUF8075, 870 m, 2-VI-2005, A.V. Pérez Latorre et al., MGC 63899.

\section{Categoría UICN: NT}

Observaciones. No citada por Valdés (1987) en Andalucía Occidental. Nuestras referencias (Pujadas Salvà et al., 1994: 135) sobre $O$. caryophyllacea Sm. para Andalucía son errores de identificación, corresponden a $O$. clausonis. Foley (2001b: 60) nombra a las plantas ibéricas como subsp. hesperina (J.A. Guim.) M.J.Y. Foley sin una clara justificación que permita diferenciarlas de la subespecie tipo (cf. Pujadas Salvà \& Lora González, 1997).

14. O. rapum-genistae Thuill., Fl. Paris, ed. 2: $317(1800)$

CÁDIZ: Serranía de Ronda: Sierra del Pinar, 1600 m, -VI-1900, E. Reverchon, WU.

Observaciones. El pliego de herbario "Elisée Reverchon- Plantes d'Espagne 1900/ Province de Granade / Sierra del Pinar sur les racines des pins, sur le calacaire, $1600 \mathrm{~m} / \mathrm{Juin} /$ WU" es algo dudoso, habría que comprobar la veracidad de la localidad citada en la etiqueta. No hemos podido encontrar ningún otro material que respalde su presencia en toda la Serranía de Ronda.

CÓRDOBA: Sierra Morena: Espiel, Peña Crispina, 319517 / 4236875, 895 m, 5-V-2007, E. Reyes Sepúlveda, COA 37645. Pozoblanco, 
Peña del Águila, 336133 / 4230563, 777 m, 15 V-2007, E. Reyes Sepúlveda, COA 37648. Alcaracejos, c. Puerto Calatraveño, 326952 / 4237099, 710 m, 22-V-2007, E. Reyes Sepúlveda, COA 37662. GRANADA: Cazorla-Segura-Las Villas: La puebla de D. Fadrique, c. del Puerto del Pinar, 30SW4313, $1700 \mathrm{~m}, 3-\mathrm{VI}-2000$, A.Pujadas \& J.A. López, COA 29576. La Puebla de D. Fadrique, pr. Cortijo de la Canaleja, 30SWH4407, 1600 m, 20-V-1978, J. Leal, MA 294121. HUELVA: Sierra de Aracena: Cumbres de San Bartolomé, Bailones, 30SPC9217, 22-IV98, M. López, COA 25302. La Nava, barranco El Helechoso, 30SPC9304, M. López, COA 25303. Arroyomolinos de León, Los Bonales, 29QC3012, 15-II-2003, E. Sánchez Gullón \& P. Weickert, COA 33983. Campiña de Huelva y CondadoAljarafe: Pinares de Hinojos, c. Casa del Membrillo, 1-VI-2001, L. Plaza, COA 33984. Litoral: c. Parador Nacional, Mazagón, Moguer, 29SPB9510, 12-IV-2001, E. Sánchez Gullón, COA 37512. Laguna de las Madres, Los Hermanillos, umbría sur de Mazagón, 29SPB8914, 28-IV-2001, E. Sánchez Gullón \& A. Pujadas, COA 31073. Palacio del Acebrón, El Mesto, Arroyo de la Rocina, Almonte, 29SQB1814, 28 IV-2001, E. Sánchez Gullón \& A. Pujadas, COA37511. Lepe, alcornocal, 29SPB5926, 21III-2002, E. Sánchez Gullón \& A. Pujadas, COA 37474. Isla Cristina, La Redondela, 20SPB5322, 21-III-2002, E. Sánchez Gullón \& A. Pujadas, COA 37510. Acantilado de Mazagón, Moguer, 29SPB9511, 20-IV-2003, E. Sánchez Gullón \& P. Weickert, COA 37513. Carretera AlmonteParador, 21-II-1982, J. Herrera et al., SEV 162943. Arroyo del Loro, 21-III-1982, F. Gómez et al., SEV 162879. JAÉN: Cazorla-Segura-Las Villas: Santiago de la Espada a Pinar Negro, 30SWH1801, 1680 m, 16-VII-1993, C. Ferrero, JAEN 935031. MÁLAGA: Sierra del Aljibe: Benarrabá, Arroyo de las Cobatillas, 30STF9646, 300-400 m, 29-V-2004, O. Gavira, MGC 61187.

Categoría UICN: NT

Observaciones. Planta rara en todo el territorio que solamente es algo frecuente a nivel local en la provincia de Huelva. Las citas de Valdés (1987: 555) en Sierra Norte de Sevilla y en Algeciras no se han podido contrastar, probablemente alguna corresponda a $O$. gracilis subsp. deludens.
15. O. gracilis Sm., Trans. Linn. Soc. London 4:172 (1798)

- Hojas superiores 3-7(9) mm de ancho, lanceoladas a ovadas, más o menos esparcidas. Brácteas 2.5-4(6) $\mathrm{mm}$ de ancho. Cáliz con venas inconspicuas. Corola amarilla o marrón-amarillento, raramente con venas reticuladas, ápice generalmente rojo externamente, rojo oscuro brillante internamente. Filamentos adaxiales insertos a (1)2-4(6) mm sobre la base de la corola, los filamentos abaxiales insertos a (0.5)1-2.5(3) $\mathrm{mm}$; filamentos ensanchados gradualmente hacia la base, pelosos en la base

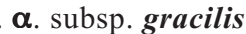

- Hojas superiores (5)8-12(16) mm de ancho, ovadas a ovado-deltoideas, densas, a veces subimbricadas. Brácteas (4)7-12 mm de ancho. Cáliz con venas conspicuas. Corola ocrácea con venas reticuladas, raramente sin venas, ápice ocráceo, raramente rojizo externamente, ocrácea o rojiza internamente. Filamentos adaxiales insertos a 1-2(3) $\mathrm{mm}$ sobre la base de la corola, los filamentos abaxiales insertos a 0.5-1.5(2) $\mathrm{mm}$; filamentos estrechos, bruscamente dilatados en la base, pubérula, a veces glabrescentes en la base

$\boldsymbol{\beta}$. subsp. deludens

\section{$\alpha$. subsp. gracilis}

Presente en Alpujarras bajas, Contraviesa y Sierra de Gádor, Sierra Nevada y Filabres, Sierras de María, Orce y Estancias, Almería Subdesértica (Pujadas Salvà \& Lora González, 1996: 218). Aportamos nuevas citas que amplían su área de distribución. (UICN: NT).

ALMERÍA: Sierras de María, Orce y Estancias: Regione montana montis Sierra de María, 4000', 12-VII-1845, M.H. Willkomm, COI-H ${ }^{\circ}$ Willkomm (sub Orobanche cruenta Bertol.; citado en Willkomm, 1870: 621). Altiplanos Manchegos y Almería Subbética: Carretera de Cañada de Cañepla a María, WG6677, J.L. Vivero \& R. Feria, COA 33964. Almería Subdesértica: $\mathrm{S}^{\mathrm{a}}$ de Lisbona, 30SWG8727, 320 m, 15-IV-2005, A. Pujadas, COA 33965. CÁDIZ: Litoral: Chiclana, La Barrosa, 28-III-2004, D. Rubiales, COA 33924. Circa Chiclana, 1859, Kalisch, COI-H ${ }^{\circ}$ Willkomm (sub Orobanche cruenta Bertol.; citado en Willkomm, 1870: 621). Valle del Guadalquivir: Morón de la Frontera a Coripe, km 7, 30-III-1997, J. Pusch, COA 23586. Sierra del Aljibe: P.N. Alcornocales, subida Pico del Aljibe desde Refugio del Picacho, 6-V-1995, A. Pujadas 
\& A. Lora, COA 17396. Algeciras, Alcornocales, Arroyo de la Miel, 19-V-2001, L. Plaza \& J. Salcedo, COA 33970. Algeciras, $\mathrm{S}^{\mathrm{a}}$ de Luna, Pista del Tío de las Norias, $600 \mathrm{~m}, 24-\mathrm{IV}-2004, A$. Pujadas et al., COA 33969. Alcalá de los Gazules, Loma de la Perejila, 23-IV-1979, B. Cabezudo et

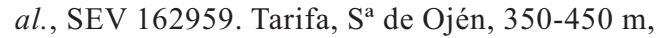
alcornocal, 16-IV-1974, S. Talavera \& B. Valdés, SEV 162899. Serranía de Ronda: El Gastor, $S^{a}$ del Gastor, TF9280, 29-V-1983, A. Aparicio et al., SEV 162958. CÓRDOBA: Sierra Morena: La Gargantilla, Sierra de Villaharta., 18-IV-1992, F. Martínez, COA 17398. Obejo, ctra. al pantano, 18-V-2001, A. Pujadas \& D. Rubiales, COA 33927. Córdoba, Las Ermitas, 30SUG3897, 460 m, 13-IV-02, A. Pujadas, COA 33921. Córdoba, Los Villares, 5-V-2003, A. Pujadas, COA 33971. Córdoba, cerca Arroyo Bejarano, 7-V-2006, A. Pujadas, COA 37499. Pedroches: Pozoblanco, Fuente La Lancha, Cortijo El Lote, 26-V-2000, J. Rubio, COA 29916. Subbética Occidental: Carcabuey, El Palancar, 16-V-2004, E. Triano \& A. Pujadas, COA 33968. GRANADA: Sierra de Mágina: Pedro Martínez, Mencal, Cañada del Tesoro, 30SVG8350, 1250 m, R. Sánchez Pérez, GDA 26244. Cazorla-Segura-Las Villas: Huéscar, La Sagra, el Canuto, 30SWH3801, 1800 m, 16VI-2002, F.B. Navarro, GDA 48712. Sierra de La Sagra. WG3699, 1700-1850 m, 24-VI-1988, B. Valdés et al., 2009, SEV s/n (en Valdés \& Talavera, 1991: 204). Depresión de Granada: Cogollos Vega, c. Río Blanco, 30SVG5228, 1150 m, M.L. Arrebola \& L. Cano, GDA 22366. Sierras de Almijara, Guájares y Trevenque: Base del Trevenque, cerca de la Cortijuela, 1700 m, 12VII-1996, A. Pujadas \& A. Lora, COA 24867. Cogollos Vega, pr. Río Blanco, 30SVG5228, 1150 m, 21-V-1988, M.L. Arrebola \& L. Cano, GDA 22366. Puerto de la Mora, 1200 m, 13-VI-1975, T. Palomeque, JAEN 630649 y 645649. Arenas del Rey, alrededores pista aterrizaje, 30SVF2587, 1000-1100 m, 3-VI-2004, B. Cabezudo et al., MGC 59651. Alhama de Granada, entre las Llanadas y las Chorreras, 30SVF1084, 1600-1750 m, 23-VI-2004, B. Cabezudo et al., MGC 58656. Depresión de Guadix-Baza y Guadiana Menor: Zújar, baños, 30SWG1661, 670 m, C. Fernández et al., JAEN 640561. Sierra Nevada y Filabres: Peñones de San Francisco, 1-VII-1990, $A$. Pujadas \& P. Poyato, COA 17402. Arroyo
Juntillas, 1-VII-2001, J. Salcedo \& L. Plaza, COA 33972. Lugros, Dehesa del Camarate, 30SVG7716, 1470 m, 6-VII-2002, L. Plaza \& A. Pujadas, COA 37631. Supra Bubión, 2-VI-1902, C. Pau, MA 435751. Fuente de San Jerónimo, 2000 m, 26-VI-1980, J.A. Devesa et al., SEV 162936. Oberer Teil der Tales der Río de Mulhacén, ca. 2500-3000 m, 8-VIII-1924, A. Ginzberger, WU s/n. Sierra de Baza: Santa Bárbara a La Canaleja, 7-VI-1983, J. Torres et al., GDAC 27137. Cerro Quintana, 30SWG1040, 1800 m, 22-VI-2002, F.B. Navarro, GDA 47705. Ascent to Santa Bárbara, WG1340, 1400-1600 m, 21-VI-1988, B. Valdés et al., Itinera 1334, SEV s/n (sub O. caryophyllacea en Valdés \& Talavera, 1991: 203). HUELVA: Sierra de Aracena: Aroche, Los Llanos, Rivera de Chanza, 30SPC7806, 6-V-1997, M. López, COA 25300. Aracena, Guadalcanar, 30STH7612, 2-VI-1997, M. López, COA 23551. Cumbres de Enmedio, Higuera la Real, Casa del Pollo, 4-III-1998, M. López, COA 24873. Cumbres de San Bartolomé, camino Valdemontoso, 11-III-1998, M. López, COA 25540. Linares de la Sierra, cerros de la Viña Vieja, 30SQB1089, 19-III-1998, M. López, COA 25541. Cumbres de San Bartolomé, $S^{\text {a }}$ del Álamo, 29SPC9913, 17-V-2005, E. Sánchez Gullón \& J.A. Ortega, COA 33966. Entre La Corte de Santa Ana y Almonáster, 12-V-1978, J. Rivera et al., SEV 62025. Campiña de Huelva y Condado-Aljarafe: San Juan del Puerto, Dehesa de la Torre, 15-III-2001, 29SPB8637, E. Sánchez Gullón, COA 33967. JAÉN: Abundantes referencias de Molino Olmedo et al. (1995) a las que añadimos las siguientes. Sierra Morena: Andújar, Sa Quintana, 30SUH9648, 1000 m, 13V-1984, 5-V-1985, E. Cano, JAEN 84391 (sub O. foetida en Molino Olmedo et al., 1995: 48). Santa Elena a La Aliseda, 11-V-1941, S. Rivas Goday \& F. Bellot, MAF 36175. Subbética Occidental: Pico de la Pandera, 22-VI-2000, B. Román \& C. Martínez, COA 29913. Sierra Mágina: Sierra de Huelma, Loma de Peña Lisa, 1000 m, 24-VI-1925, Cuatrecasas, BC 46587 \& MAF 36204 (sub var. spruneri en Cuatrecasas, 1929: 429). Mágina Oriental, hacia Caño del Aguadero, 1800 m, 30-VI-1925, Cuatrecasas, BC 46589 (sub var. spruneri en Cuatrecasas, 1929: 429). Serrate, 20-VII-1925, Cuatrecasas, BC 46578 (sub var. spruneri en Cuatrecasas, 1929: 
429). Cárceles, $1400 \mathrm{~m}, 17-\mathrm{VI}-1926$, Cuatrecasas, BC 46588 \& MA 435796 (sub var. glabra en Cuatrecasas, 1929: 429). CazorlaSegura-Las Villas: Sierra de Segura, Pto. Lezar, 30SWH2103, 1-VII-1997, P. Prados, COA 17595. Hornos, pantano del Tranco, 27-VI-1979, T. Palomeque, JAEN 630681. Sierra Cabrilla, Barranco de Gines, 25-VI-1980, 1770-2000 m, J.A. Devesa et al., SEV 162932. Sierra Seca, E1 Chaparral, WH2800, 1800 m, 29-VI-1988, B. Valdés et al., SEV (sub O. rapum-genistae en Valdés \& Talavera, 1991: 205). Cortijos Nuevos, El Yelmo, 1900 m, Aparicio et al., SEV 162907. MÁLAGA: Sierra del Aljibe: Júzcar, hacia los Riscos, UF0557, 900 m, 30-IV-2006, O. Gavira, MGC 64769. Serranía de Ronda: Cañete La Real, 4-V-1985, M. Ruiz, COA 17401. Pto. de Montejaque, dirección Grazalema, 30STF9774, 20-IV-1995, A. Pujadas, COA 17399. Ardales, alrededores de casa forestal y Fuente Carrizo, 30SUF8840, 15-V-1997, L. Plaza et al., COA 23362. Antequera, cara N Sierra Huma, 30SUF4488, 6-VI-1997, L. Plaza et al., COA 24144. Campillos, Sierra de Peñarrubia, La Herriza, 30SUF3592, 400 m, 26-III-1998, $A$. Pujadas et al., COA 25553. Sierra Blanca, c. Ojén, 7-V-1982, Merino, MGC 11783. Axarquía: Pantano de la Concepción, UF24, III-1977, Zarazaga, MGC 5504.

及. subsp. deludens (Beck) A. Pujadas, Lagascalia 26: 247 (2006)

Orobanche haenseleri var. deludens Beck, Repert. Spec. Nov. Regni Veg. 18: 38 (1922) [basión.]

O. gracilis var. deludens (Beck) A. Pujadas, Anales Jard. Bot. Madrid 61(2): 131 (2004)

O. austrohispanica M.J.Y. Foley, Anales Jard. Bot. Madrid 58(2): 224 (2001)

$O$. reticulata auct. hisp., non Wallr., Orob. Gen.: 42 (1825)

O. gracilis var. spruneri auct. hisp., non Orobanche spruneri F.W. Schultz in Flora (Regensburg) 26: 130 (1843)

Presente en Litoral, Valle del Guadalquivir, Subbética Occidental, Sierra Mágina, Sierra del Aljibe, Serranía de Ronda, Axarquía, Sierras de Almijara, Guájares y Trevenque, Alpujarras bajas, Contraviesa y Sierra de Gádor, Sierra Nevada y Filabres, Almería Subdesértica (Pujadas Salvà \&
Lora González, 1996: 218, sub. var. spruneri; Pujadas Salvà \& Plaza Arregui, 2004, sub var. deludens). Aportamos nuevas citas que amplían su área de distribución. (UICN: NT).

ALMERÍA: Almería Subdesértica: San José, Sierra del Cabo de Gata, Barranco del Sabinar, WF76, 200-350 m, 2-VI-1967, P.W. Ball et al., ABH 32918. CÁDIZ: Valle del Guadalquivir: Puerto de Guillén ditionis Jerez, 9-V-1890, Pérez Lara, MAF 36174. Sierra del Aljibe: Los Alcornocales, nacimiento del Alberite, $36^{\circ} 27^{\prime} 12.4^{\prime \prime} \mathrm{N}$; $5^{\circ} 35^{\prime} 23.8^{\prime \prime} \mathrm{W}, 22$ IV-2002, L. Plaza \& J. Salcedo, COA 37496. Sierra Palma p. Algeciras, 19-IV-1876, M. Winkler, COI- $\mathrm{H}^{\circ}$ Willkomm (subOrobanche rapum Thuill.; citado en Willkomm, 1893: 621, como Orobanche Rapum Genistae [sic.] Thuill.). Serranía de Ronda: P.N. Grazalema, Pico San Cristóbal, vertiente norte, $1300 \mathrm{~m}, 20-\mathrm{V}-1995, A$. Lora et al., COA 37493. P.N. de Grazalema, S del Endrinal, 30STF8769, 1300 m, 21-VI-1995, A. Lora et al., COA 37621. Sierra del Peralto, Camino de Villaluenga a Los Llanos del Republicano, Casa Cabeza Caballo, 20-IV-2002, D. Rubiales, COA 37494. Grazalema, Río Gaduares, alcornocal adehesado, L. Plaza, 21-IV2002, COA 37495. Algodonales, Puerto de la Vieja, TF8984, 500 m, 30-IV-1984, A. Aparicio \& J.G. Rowe, SEV 115450. GRANADA: Subbética Occidental: Sierra de Madrid, próx. Cortijo del Cañuelo, VG2130, 1100 m, 15-VI1983, P. Aroza et al., GDA 16552. Serranía de Ronda: Entre Alhama de Granada y Loja, 4102173 / 408627, $1000 \mathrm{~m}, 30-\mathrm{V}-2003, C$. Burgarella, COA 37497. Sierras de Almijara, Guájares y Trevenque: Alhama de Granada, S ${ }^{a}$ Tejeda, hacia la Maroma, 30SVF1186, $1100 \mathrm{~m}$, 15-VI-2000, C. Morales et al., GDA 43757. Trevenque, La Cortijuela, 10-VI-1975, T. Palomeque, JAEN 630638. Trevenque, Puente de Siete Ojos, 17-VI-1978, T. Palomeque, JAEN 630636. Sa de Alfacar, Huétor-Santillán, Fuente de La Teja, 30SW5524, 1340 m, 20-VI-1922, Aedo et al., MA 508636. Almuñécar, subida a Cerro Gigantes, 30SUF3074, 900-1000 m, 29-IV2004, B. Cabezudo et al., MGC 59653. Alpujarras bajas, Contraviesa y Sierra de Gádor: Cuenca del Beniamar, VF9995, c. Laroles, 18-VI-1984, J. Guirado, GDAC 28829. JAÉN: Subbética Occidental: Jaén a Puente de la Sierra, km 10, 
VG37, 500 m, 8-V-1973, C. Fernández, JAEN 4727 (sub O. caryophyllacea, en Molino Olmedo et al. 1995: 48). Valdepeñas de Jaén, La Pandera, VG26, 1500 m, 27-VI-1977, C. Fernández López, JAEN 771786. Mingo, 27-V-1978, T. Palomeque, JAEN 630669. Los Villares, La Pandera, VG26, 1450 m, 4-VII-1980, C. Fernández López, JAEN 801074. Los Villares, Río Frío, 30SVG2968, 24V-1989, C. Fernández et al., JAEN 913088 (sub O.amethystea en Molino Olmedo et al., 1995: 47). Sierra Mágina: Sierra de Cabra del Sto. Cristo, 4-VI-1925, Cuatrecasas, BC 46625. Cambil, La Mata, 1050 m, 18-IV-1981, C. Fernández, JAEN 81415 (sub O. caryophyllaceae, en Molino Olmedo et al., 1995: 48). Loma de Peña Lisa, 1000 m, 24-VI-1925, Cuatrecasas, MAF 36204 (sub $O$. gracilis var. spruneri). MÁLAGA: Sierra del Aljibe: Faraján, carretera Júznar-Faraján, 30SUF0555, 700 m, 22-III-1987, D. Montilla, MGC 40472. Benarrabá, Los Pepes, 30STF9744, 200 m, 8-IV-2004, O. Gavira, MGC 61222. Gaucín, La Almuña, 17-IV-2004, J.A. García Rojas, MGC 63055. Jubrique, carretera hacia Estepa, Arroyo de las Aguzaderas, 30SUF0548, 800 m, 5-VI-2004, O. Gavira, MGC 61206. Serranía de Ronda: Sierra Blanca, Ojén, 15-V1992, M.C. Balaguer, COA 17632. Cerca del Cerro de los Enamorados, 30SVF2064, $1700 \mathrm{~m}$, 18-V-1996, A. Lora \& L. Plaza, COA 22096. Coin, Sierra Alpujata, 22-III-1987, E. Cano \& S. Heville, MGC 23734. Tolox, Sierra de Tolox, subida a cerro Corona, 30-V-1996, P. Navas et al., MGC 53784. Istán, Sierra Real, pasado Puerto de la Refriega, 7-VI-1996, Y. Gil et al., MGC 46062. Axarquía: Montes de Málaga, UF8176, 23-III-1990, T. Sánchez \& J. Vera, MGC 30457. Montes de Málaga, ctra. de Comares, 30SUF8075, 800 m, 2-VI-2005, F. Soriguer et al., MGC 63449. Sierras de Almijara, Guájares y Trevenque: Sierra de Almijara, Canillas de Albaida, VF08, 19-IV-1982, B. Cabezudo et al., MGC. Cómpeta, Sierra Almijara, 8-V-1996, $P$. Navas et al., MGC 44650. Cómpeta, carril de puerto Collado a Casa Mina, VF1576, 850-900 m, 1-IV-2003, B. Cabezudo et al., MGC 59649. Canillas de Albaida, Arroyo de la Cueva del Melero, VF1480, 690 m, 2-V-2003, B. Cabezudo et al., MGC 59648. Frigiliana, Venta Panaderos, Río Higuerón, VF2078, 740 m, 23-V-2003, B. Cabezudo et al., MGC 61237.
Observaciones. Las referencias de $O$. gracilis var. spruneri en Pujadas Salvà \& Lora González (1996: 218) y en Pujadas Salvà (2002: 431-432) corresponden a este taxón. Foley (2001a) describió esta planta como $O$. austrohispanica, pero con el rango taxonómico a nivel específico el nombre correcto es el de $O$. deludens (Beck) A. Pujadas, comb. et stat. nov. [O. haenseleri var. deludens Beck, Repert. Spec. Nov. Regni Veg. 18: 38 (1922) (basión.)]

Las referencias de $O$. reticulata Wallr. en Valdés (1987: 552) corresponden en parte a $O$. gracilis subsp. deludens.

16. O. foetida Poir., Voy. Barbarie 2: 195 (1789) - Planta hasta $70 \mathrm{~cm}$ de altura, tallo robusto. Corola grande, hasta $22 \mathrm{~mm}$. Filamentos adaxiales insertos a 2,5-4,5 $\mathrm{mm}$ sobre la base de la corola, los filamentos abaxiales insertos a $1,5-3 \mathrm{~mm}$ $\alpha$. var. foetida Planta menor hasta $35 \mathrm{~cm}$ de altura, tallo delgado. Corola menor, hasta $17 \mathrm{~mm}$. Filamentos adaxiales insertos a 1,5-3 mm sobre la base de la corola, los filamentos abaxiales insertos a 0,5-2 $\mathrm{mm}$

及. var. broteri

Observaciones. O. foetida es un taxón de morfología muy variable del que reconocemos estas dos variedades que a veces son difíciles de diferenciar ya que se pueden encontrar numerosas formas intermedias.

\section{$\alpha$. var. foetida}

Presente en Sierra de Aracena, Sierra Morena, Pedroches, Campiña de Huelva y Condado-Aljarafe, Litoral, Valle del Guadalquivir, Sierras de Almijara, Guájares y Trevenque (Pujadas Salvà et al., 2003: 740-741). Aportamos nuevas citas que amplían o confirman su área de distribución.

CÁDIZ: Litoral: Caño río San Pedro, Puerto Real, 3-V-2003, D. Rubiales, COA 37521. Estuario Arroyo San Ambrosio, Caños de Meca, 20-II-2002, E. Sánchez Gullón \& P. Weickert, COA 37523. Rozas de Puerto Real, 17-VI-1986, F.G. Manzaneque, MA 556907. De Conil de la Frontera a Cabo Roche, 29SQA52, 26-V-1988, A. Izuzquiza et al., MA 430691. El Español prope Jimena, 30-V-1881, Pérez Lara, MAF 36196. CÓRDOBA: Sierra Morena: Embalse del Guadalmellato al de San Rafael de Matallana, 27- 
IV-2003, A. Moral, COA 37528. Valle del Guadalmellato, ctra. Embalse a la altura del Arroyo de S. Antón, 25-IV-1980, M. Arenas \& E. Dominguez, COFC 6544. Pedroches: Pozoblanco, E1 Majago, 30SUH4225, 23 -III-2001, J.L. Sánchez Sánchez, COA 31054. Sendero de la Vegueta, Cardeña, 23-IV-2006, L. Plaza et al., COA 37526. Valle del Guadalquivir: Montoro, E1 Piruetenar, Loma de la Marquesa, 30SUH9215, 220 m, 13-VII-1991, E. Cano, JAEN 990415. HUELVA: Sierra de Aracena: Cortegana, 28-VI1988, Ladero \& Amor, SALA 79728. Entre Aracena y Corterrángel, 18-VI-1978, J. Rivera, SEV 62024. Andévalo: Gibraleón, El Campillo, 29SPB7742, 15-IV-2003, E. Sánchez Gullón \& P. Weickert, COA 37525. Calañas, 23-V-2003, E. Sánchez Gullón \& P. Prendas, COA 37523. Entre Calañas y Puentes del Río Odiel, 4-V-1985, $P$. Weickert, SEV 162944. Campiña de Huelva y Condado-Aljarafe: Los Medios, 29SPB7528, 16IV-2003, E. Sánchez Gullón \& P. Weickert, COA 37524. Entre Valverde del Camino y La Palma del Condado, 17-IV-1966, A. Sánchez Jurado, MAF 75196. Litoral: Justa Huelva, 2-V-1903, C. Pau, MPU-H ${ }^{\circ}$ Coste. JAÉN: Citas de Molino Olmedo et al. (1995) a las que añadimos las siguientes. Sierra Morena: Sierra de Andújar, pr. Santuario Virgen de la Cabeza, 23-V-2004, A. Pujadas, COA 37527. Entre Andújar y el Santuario de N.S. de la Cabeza, Lugar Nuevo, 13-IV-1988, J. Herrera et al., SEV 162919. Subbética Occidental: Carretera de Córdoba Km 2, VG28, 600 m, 7-V-1973, C. Fernández López, JAEN 73417. Puente de la Sierra, Silla Reina, VG37, 600 m, 15-VI-1976, C. Fernández López, JAEN 1207076. MÁLAGA: Sierra del Aljibe: Estepona, San Diego, 40 m, 20-V-1979, T. Palomeque, JAEN 630686. Gaucín, cruce del Colmenar, 30STF8943, 640 m, 29-V-2004, O. Gavira, MGC 60746. Serranía de Ronda: Istán, P.N. Sierra de Las Nieves, Monte de Boorque, 30SUF25, 9-V-2000, Y. Gil et al. MGC 50369. Ronda, 3-VI-1890, E. Reverchon, PRC, W 4333 \& WU. Axarquía: Prope urbem Málaga, 1844, Prolongo, COI-H ${ }^{\circ}$ Willkomm (citado en Willkomm, 1870: 622). Prope pagum Fuengirola, 18-IV-1845, H.M. Willkomm, COI-H ${ }^{\circ}$ Willkomm (citado en Willkomm, 1870: 622). Vélez-Málaga, Almayate, El Peñón del Toro, 30SVF0049, 17IV-2004, J. García-Sánchez \& R. Torreblanca,
MGC 64804. Pizarra, Hacho de Pizarra, subida al Santo, 30SUF4869, $190 \mathrm{~m}, 30-\mathrm{III}-2006, A$. Pérez Latorre et al., MGC 64774. Loc. erectis Sierrae de Alora, 3-V-1879, Huter, Porta \& Rigo, $n^{\circ} 854$, PRC. Sierras de Almijara, Guájares y Trevenque: Collibus inter pages Canillas de Aceituno, Sedella, Cómpeta et Frigiliana papium, 11-VI-1845, Willkomm, COI-H ${ }^{\circ}$ Willkomm (citado en Willkomm, 1870: 622). Canillas de Albaida, 9-VI-1935, M. Laza Palacios, MAF 36199. SEVILLA: Sierra Morena: Almadén de la Plata, 5-IV-2002, L. Plaza \& J. Salcedo, COA 33963. Vega del Guadalquivir: Prope Dos Hermanas, 21-IV-1895, C. Pau, BC 46645. Valle del Guadalquivir: El Gandul, 24-IV-1979, P. Candau et al., SEV 162888.

Categoría UICN: LC.

Observaciones. La referencia "Las Rozas de Puerto Real, JACA 73484" corresponde a una localidad de la provincia de Cádiz y no de la provincia de Madrid como se indicó por error en Pujadas Salvà et al. (2003: 741).

ß. var. broteri (J.A. Guim.) Merino, Fl. Galicia 2: 49 (1906)

Orobanche crinita var. occidentalis M.J.Y. Foley, Anales Jard. Bot. Madrid 58(2): 226 (2001)

O. foetida lusitanica Brot., Phytogr. Lusit. 2: 149 (1827)

O. foetida subsp. broteri J.A.Guim., Broteria 3:105 (1903) [basión.]

O. foetida subsp. lusitanica Cout., Fl. Portugal 568 (1913)

O. foetida var. lusitanica (Cout.) Beck in Engl., Planzenr. 96: 295 (1930)

O. sanguinea auct., non C. Presl, Del. Prag. $71(1822)$

Presente en Litoral, Valle del Guadalquivir, Alpujarras bajas, Contraviesa y Sierra de Gádor, Sierra Nevada y Filabres (Pujadas Salvà \& Lora González, 1996: 219, sub subsp. broteri; Pujadas Salvà et al., 2003: 742-743). Aportamos nuevas citas que amplían su área de distribución.

ALMERÍA: Sierra Nevada y Filabres: Paterna del Río, Fuente Agria, 1200 m, 28-VII2007, L. Posadas \& A. Ivorra, COA37657. CÁDIZ: Litoral: Puerto de Santa María, 9-IV1977, T. Palomeque, JAEN 630700. Vejer de la Frontera, Los Caños de Meca, 27-IV-1978, T. 
Luque et al., SEV 162898. Sierra del Aljibe: Algeciras a Tarifa, 18-V-1978, T. Palomeque, JAEN 630654. HUELVA: Sierra de Aracena: Entre Galaroza y Los Marines, 9-VII-1969, S. Silvestre, SEV 162875. Litoral: Ayamonte, 9-V1943, C. Vicioso, MA 115177. MÁLAGA: Serranía de Ronda. Fuengirola a Marbella, $20 \mathrm{~m}$, 23-V-1976, T. Palomeque, JAEN 630623.

Categoría UICN: NT

Observaciones. Valdés (1987: 555) cita $O$. foetida sólo a nivel específico. Las referencias de Foley (2001a: 226; 2001b: 70-71) de O. crinita var. occidentalis corresponden a $O$. foetida var. broteri (cf. Pujadas Salvà et al. 2003). También corresponden a la var. broteri muchas de las citas de O. sanguinea para la Península Ibérica (cf. Pujadas Salvà et al. 2003 ).

17. O. densiflora Reut. in A. DC., Prodr. 11: 19 (1847)

Es una especie bien caracterizada, abundante en la costa de Cádiz. Aportamos varias citas que abarcan toda su área de distribución en Andalucía y probablemente en toda la Península Ibérica ya que las referencias de Portugal no se han podido contrastar.

CÁDIZ: Litoral: Rota, Punta Candor, 29SQA3257, 26-IV-1998, A. Pujadas, COA 25299. Barbate, Pinar de la Breña, 17-II-2002, E. Sánchez Gullón \& P. Weickert, COA 33977. Conil, Fuente del Gallo, 19-V-2002, A. Pujadas, COA 33974. Chiclana, Sancti Petri, 15-III-2003, A. Pujadas, COA 33975. Barbate, Punta de Trafalgar, 15-V-1959, D.M.C. Brinton-Lee, SEV 162880. Entre El Puerto de Santa María y Fuentebravía, 27-IV-1978, T. Luque et al., SEV 162906. Sierra del Aljibe: Tarifa, Playa de Bolonia, 29-VI-1986, A. Pujadas \& P. Poyato, COA 13890. La Línea, junto al aeropuerto, 11V-1988, J. Oliveira, COA 13887. Zahara de los Atunes, Playa de la Atlanterra, 10-V-2003, A. Pujadas, COA 33976. Algeciras, Palmones in Loto cretico et Medicago marina, 10-V-1873, $R$. Fritze, MAF 36183 \& COI-H ${ }^{\circ}$ Willkomm (citado en Willkomm, 1893: 187). Tarifa, Punta Paloma, 28-IV-1978, T. Luque et al., SEV 162904.

Categoría UICN: VU [B1ac(iv)]

Observaciones. Existe un pliego de $O$. densiflora para la provincia de Jaén "Carchelejo, Sta. Lucía, 780 m, 8-VI-1987, C. Fernádez López,
JAEN 771372", que debe de corresponder a un error de etiqueta. En la zona no hay ninguna población de esta especie típica de dunas costeras, además el pliego testigo conserva granos de arena de playa, arena que no se puede encontrar en la provincia de Jaén. Willkomm (1870: 622) la citó también en Jaén basado en una referencia de Lange, creemos que se trata de un error de identificación.

18. O. haenseleri Reut. in DC., Prodr. 11:22 (1847)

Orobanche hellebori Miégeville, Bull. Soc. Bot. Fr., $12: 347$ (1866)

Presente en Alpujarras bajas, Contraviesa y Sierra de Gádor, Sierra Nevada y Filabres (Pujadas Salvà \& Lora González, 1996: 212). Aportamos nuevas citas que amplían su área de distribución.

ALMERÍA: Sierra Nevada y Filabres: $S^{a}$ de los Filabres, Barranco del Negro, 30SWG4021, 1810 m, 4-VII-1997, J. Peñas et al., HUAL 3745. GRANADA: Sierras de Almijara, Guájares y Trevenque: Nevada, Mts. Dornajo, 20-VII-1873, M. Winkler, COI. Dílar, río Dílar, próximo a Barranco Hondo, 30SVG6002, 1980 m, 23-VI2003, J. Lorite et al., GDA 49336. Dornajo, 22VII-1892, A.E. Lomax, MA 114975. Sierra Nevada y Filabres: Trevélez, cerca del Cortijo Calvache, 16-VII-1990, A. Pujadas \& P. Poyato, COA 16495. Dólar, umbría Sierra Nevada, 30SWG0010, $1700 \mathrm{~m}, 21-\mathrm{VI}-1994$, A. Pujadas \& A. Pallarés, COA 16493. Sierra Nevada. Barranco de los Alisos, afluente del río Dúrcal, entre abedules, 1800 m, 30-VI-1997, J.L. Vivero, COA 13582. Lugros, Dehesa del Camarate, 30SVG7716, 1470 m, 19-VI-2003, A. Pujadas \& L. Plaza, COA 33980. Oberer Teil des Tales des Río Mulhacén, c. 2500-3000 m, 8-VIII-1924, Ginzberger, WU 2820. JAÉN: Sierra de Mágina: Almadén, Cabezaprieta, 1200 m, 11-VI-1926, Cuatrecasas, BC 46635 (citado en Cuatrecasas, 1929: 429). Cárceles, 1800 m, 5-VII-1926, Cuatrecasas, BC 46652 (sub O. major vidit Pau, en Cuatrecasas, 1929: 430). El Almadén, 30SVG5373, 1800m, 13-VII-1997, A. Pujadas, COA 23553. Sierra de Mágina, El Almadén, Torres, 16-VI-2000, B. Román \& C. Martínez, COA 29921. Torres, Cerro Cárceles, 1800 m, 11VII-2006, A. Pujadas et al., COA 37607. Cazorla- 
Segura-Las Villas: Sierra de Cazorla, ruta desde el Empalme del Valle hacia la fuente del Oso, 30SWG5041, 17-VII-1997, L.F. Perea \& J. Madrigal, COA 12571. Sierra de Cazorla, empalme del Valle, 30SWG5041, 17-VII-1997, L.F. Perea \& J. Madrigal, COA 12680. Sierra de Cazorla, Tranzón de Navahonda, 30SWG5041, 18-VII-1997, L.F. Perea \& J. Madrigal, COA 9229. Sierra de Cazorla, Collado de los Pegueros, 30SWG5041, 19-VII-1997, L.F. Perea \& J. Madrigal, COA 12569. Sierra del Pozo, Cortijo del Poyo Manquillo, 15-VIII-1996, L. Plaza, COA 13495. Santiago de la Espada, Km 2,5 a Pontones, 17-V-2000, A. Pujadas, COA 29920. Sierra Cazorla, Arroyo de Valdetrillo, 25-IV2002, L. Plaza, COA 33978. Sierra de Cazorla, Arroyo Frío, 29WG0699, VIII-2005, E. Sánchez Gullón, COA 33979. Santiago de la Espada, Los Puertos, 1500 m, 21-VI-1980, C. Fernández López, JAEN 80888 \& JAEN 80889. Santo Tomé, Chorro Palomera, 1200 m, 30SWH0508, 27-VI1993, J. Fernández Liencres, JAEN 935184 (sub O.amethystea en Molino Olmedo et al., 1995: 47). Cazorla, riachuelo en Nava de San Pedro, 30SWG1093, 1300 m, 27-VI-1997, Fernández Ocaña \& E. Fernández Prieto, JAEN 970405. Sierra Guillimona, gorge of Torilla, WH3812, 1400-1500 m, 23-VI-1988, B. Valdés et al., Itinera 1667, SEV s/n (sub O. cf. lutea en Valdés \& Talavera, 1991: 205). $S^{\text {a }}$ de Segura, Poyotello, gorge of river Segura, WH3222, 1400-1450 m, 26-VI-1988, B. Valdés et al., Itinera 2365, SEV s/n (sub O. caryophyllacea en Valdés \& Talavera, 1991: 203). Sa del Pozo, Nava de San Pablo, WG0997, c. $1450 \mathrm{~m}, 30-\mathrm{VI}-1988$, B. Valdés et al., Itinera 1687, SEV s/n (sub O. lutea Baumg en Valdés \& Talavera, 1991: 204). MÁLAGA: Serranía de Ronda: Sierra de las Nieves, El Corona, Tolox, 27-VIII-1997, L. Plaza, COA 25719. Yunquera, Tajo de la Caina, 28-VIII-1997, L. Plaza, COA 25720. Sierra de las Nieves, VF1961, 1700 m, 17-VI-1986, Bayón et al., MA 436918. Sierra de las Nieves, Puerto de los Pilones, Ronda, 30SUF1962, 1730 m, 25-VI1993, C. Aedo et al., MA 526194. Yunquera, Sierra de las Nieves, pinsapar plataforma alta, 1400 m, 4-VII-1991, B. Cabezudo et al., MGC 34217. Tolox, P.N. Sierra de las Nieves, vereda al Torrecilla, 30SUF2163, 1720 m, 12-VI-1996, Pérez Latorre et al., MGC 46059. Junquera, P.N.
Sierra de las Nieves, entre Cañada de los Hornillos y El Peñón de los Enamorados, 30SUF2265, 1350-1750 m, 18-VI-1998, $B$. Cabezudo et al., MGC 46782. El Burgo, subida a Sierra Prieta, cara norte, $1180 \mathrm{~m}$, B. Cabezudo, MGC 61949.

\section{Categoría UICN: NT}

Observaciones. Presente en las sierras de Andalucía Oriental. No hemos podido encontrar ningún testimonio que respalde las citas de Valdés (1987: 552) para Andalucía Occidental.

La localidad de la recolección "Jaén, alrededores, 600 m, JAEN 630683" es dudosa por encontrarse a muy baja altura y en un sector, Valle del Guadalquivir o Subbética Occidental, donde no se ha vuelto a encontrar. Probablemente se trate de un error de etiqueta.

\section{O. alba Willd., Sp. Pl. 3: 450 (1800)}

Presente en Sierras de María, Orce y Estancias (Pujadas Salvà \& Lora González, 1996: 212). Aportamos nuevas citas que amplían su área de distribución.

Es una planta de montaña, rara de la que hay muy pocas referencias. Se encuentra en las Sierras de Andalucía Oriental.

ALMERÍA: Alpujarras bajas, Contraviesa y Sierra de Gádor: $\mathrm{S}^{\mathrm{a}}$ de Gádor, Fondón, Llanos de Boliches, WF1786, 1650 m, 18-VI-1988, $B$. Valdés et al., Itinera 833, SEV s/n (sub $O$. amethystea en Valdés \& Talavera, 1991: 203). Sierra Nevada y Filabres: $S^{a}$ de los Filabres, próximo Piedras de Juan Machado, 30SWG3518, 1920 m, 29-VI-1993, J. Peñas, HUAL 6910. Altiplanos Manchegos y Almería Subbética: Vélez Blanco, de El Gabar a Vélez Blanco, 30S0578448 / 4174018, 1030 m, 26-IV-2007, $A$. Pujadas \& A. Ivorra, COA 37659. GRANADA: Cazorla-Segura-Las Villas : $S^{\mathrm{a}}$ de Guillimona, on the ascent of Cuerda de Mirabetes, 1000-1700 m, 23-VI-1988, B. Valdés et al., Itinera 1843, SEV $\mathrm{s} / \mathrm{n}$ (sub $O$. reticulata Wallr. en Valdés \& Talavera, 1991: 205). Sa de Guillimona, Cuerda de Mirabetes, 1800 m, 23-VI-1988, B. Valdés et al., Itinera 1880, SEV s/n (en Valdés \& Talavera, 1991: 203). Sierra Nevada y Filabres: Sierra Nevada, Barranco de San Juan, 7-VII-1987, C. Gálvez, COA 17324. Sierra Nevada, Peñones San Francisco, 1-VII-1990, A. Pujadas \& P. Poyato, COA 17326. Nigüelas, pista barranco de los 
Alisos, 30SVF59, 22-VII-1997, P. Prados et al., COA 17635. Sierra Nevada, Refugio de Piedra Partida, 30-VI-2001, J. Gil et al., COA 33952. JAÉN: Subbética Occidental: Valdepeñas de Jaén, Piedra del Agua, 1300 m, 10-VI-1977, C. Fernández López, JAEN 771487. Valdepeñas de Jaén, La Pandera, 1450 m, 20-VI-1977, C. Fernández López, JAEN 771795. La Mella, 30SVG2779, 1000 m, 23-VI-1983, C. Fernández, JAEN 832446 (citadas por Molino Olmedo et al., 1995: 47, y contrastadas). Sierra Mágina: Barranco del Morbote, versus Albánchez, 1150 m, 15-VI-1926, Cuatrecasas, BC 46623. E1 Almadén, 30SVG5373, 1800 m, 13-VI-1997, A. Pujadas, COA 23546. Torres, Base de Monteagudo, 30SVG5780, 1100 m, 4-VII-1998, A. Pujadas \& L. Plaza, COA 33998.

Categoría UICN: VU [B1ac(iv); D1]

Observaciones. - Citado por Foley (2001b:

52) en "Flora iberica" para Córdoba, sin embargo, el testimonio que ha dado lugar a la cita, "Córdoba, Río Guadiato, Mesas Altas y Cerro del Caballo, 3-V-1979, J. Varela, MA 294116", corresponde a $O$. crenata, por lo que se trata de un error de identificación. Además las condiciones ambientales -montañas de baja altura, temperaturas elevadas en verano-no son las adecuadas para el desarrollo de $O$. alba.

20. O. icterica Pau, Not. Bot. Fl. Españ. 3: 5 (1889)

Presente en la Subbética Occidental (Pujadas Salvà et al., 2005: 123). Aportamos nuevas citas que amplían y confirman su área de distribución.

ALMERÍA: Altiplanos Manchegos y Almería Subbética: Vélez Blanco, El Gabar, 30S0575595 / 4177790, 1056 m, 26-IV-2007, A. Pujadas \& A. Ivorra, COA 37655. Vélez Blanco, base Peña de los Letreros, 30S0579798/ 4170541, 1030 m, 26-IV-2007, A. Pujadas \& A. Ivorra, COA 37658. CÓRDOBA: Subbética Occidental: Cabra, Sierra de Cabra, 6-IX-2005, E. Triano \& A. Pujadas, COA 37514. Priego de Córdoba, Sierra de Pollos o de Jaula, 2-V-1980, J. Muñoz, SEV 162987.

Categoría UICN: VU [D1+2]

Observaciones. Las imágenes de Ivorra (2006) identificadas como O. elatior de Sierra María, Almería, corresponden a este taxón. Sin embargo, las posibles referencias bibliográficas de Cuatrecasas en Sierra Mágina de O. major han resultado corresponder a $O$. haenseleri (Cárceles, 1800 m, 5-VII-1926, Cuatrecasas, BC 46652, sub O. major vidit Pau). Schneeweiss et al. (2004, suplementary data: 4) citan O. elatior entre Martos y Jamilena (Jaén), pero el material de referencia supuestamente depositado en GDAC no se ha podido localizar. Se ha buscado, además, en dicha localidad sin éxito.

Tratada como endémica de la Península Ibérica en Pujadas Salvà et al. (2005: 121). Hemos podido estudiar recolecciones del norte de África [TÚNEZ: Makhtar to Siliana, 29-IV1990, P. Wilkin \& E.I. Wellens, RNG. Kef el Gafia, road Kairouan to Makhtar, 3-V-1975, Davis \& Lamond, RNG] por lo que hay que considerarla como endemismo ibero-africano. Además, su presencia es muy probable en Marruecos y Argelia.

Foley (2001b: 71-72) la incluye como sinónimo de $O$. elatior Sutton con un interrogante (?), sin tener en cuenta los antecedentes sobre esta especie (cf. Pujadas Salvà, 1999).

\section{O. crenata Forssk., Fl. Aegypt. 113 (1775)}

Orobanche clementei Vicioso, Anales Jard. Bot. Madrid 6(2): 75 (1946)

Presente en Alpujarras bajas, Contraviesa y Sierra de Gádor, Sierras de María, Orce y Estancias, Almería Subdesértica (Pujadas Salvà \& Lora González, 1996). Aportamos nuevas citas que amplían su área de distribución. (UICN: NT).

ALMERÍA: Almería Subdesértica: Punta Entinas, 8-IV-1954, H. Jerónimo, ALME 1722 (sub O. amethystea en Pujadas \& Lora: 213). Las Salinas, Playa de Cerrillos, 10-IV-1978, Díaz et al., COFC 13036. CÁDIZ: Litoral: Véjer de la Frontera, Finca de la Muela, 15-IV-1988, E. Morillo, COA 13529. Conil, dunas, 21-IV-1990, A. Pujadas, COA 17624. Zahara de los Atunes, Playa Atlantera, dunas, 10-V-2003, A. Pujadas, COA 37567. Entre Conil y Vejer de la Frontera, 27-IV-1978, T. Luque et al., SEV 162905. Chiclana, salinas, 21-IV-1980, F. Amor \& A. Barroso, SEV 120720 (sub O. reticulata). Valle del Guadalquivir: Jerez de la Frontera, 30-III1987, J. Oliveira, COA 13717. Ctra. BornosEspera Km 2, margen izq., 30STF5282, 18-VI1996, A. Lora et al., COA 22064. Villamartín, 
17-V-1979, P. Candau \& I. Fernández, SEV 162894. Entre Arcos de la Frontera y el Bosque, 24-V-1981, A. Martínez, SEV 120066. Entre Jerez y Torre Melgarejo, 6-III-1982, A. Martínez, SEV 121792. Trebujena, 7-V-1982, S. Talavera \& B. Valdés, SEV 120721. Sierra del Aljibe: Los Barrios a Cádiz, Km 7, 20-IV-1988, J. Oliveira, COA 13845. Algeciras, Punta del Rinconcillo, 4V-1991, M. Cuesta, COA 17615. Los Barrios, Arroyo Tiradero, TF 7008, 26-VI-1988, J. Cuenca \& S. Pérez, MGC 19316. San Roque, E1 Almendral, -IV-1959, D.M.C. Brinton-Lee, SEV 82572. Algeciras, Sierra del Algarrobo, 23-IV1980, J. Arroyo \& J.M. Gil, SEV 71579 (sub O. reticulata). Serranía de Ronda: Benamahoma, 15IV-1988, F. Sancho, COA 13723. Alrededores de Grazalema, 30STF9072, 20-IV-1995, A. Pujadas, COA 17507. Prado del Rey, 4-VI-1978, A. Ramos, SEV 162922. Algodonales, Sierra de Líjar, 29III-1980, A. Aparicio, SEV 162957 (sub O. reticulata). Benamahoma a Grazalema, Km $37-$ 38, 20-V-1983, A. Aparicio \& J. Blázquez, SEV 115473 (sub $O$. reticulata). CÓRDOBA: Sierra Morena: Hornachuelos-Palma del Río, 8-VI1983, A. Pujadas, COA 7349. Alcolea, c. del Pantano Guadalmellato, 28-IV-1985, A. Pujadas, COA 9408. Villa del Río a Cardeña, Finca Verdizales, 24-V-1989, J. Oliveira \& A. Pujadas, COA 13546. Córdoba, Las Jaras, 7-VI-1987, $A$. Pujadas \& P. Poyato, COA 13864. Arroyo Bejarano, 7-V-2006, A. Pujadas, COA 37571. Cerca del Pantano de San Rafael de Matallana, 30SUH0256, 13-IV-1997, A. Pujadas, COA 23740. Pedroches: Cardeña, Km 5 hacia el sur, 10-VI-1984, A. Pujadas \& P. Poyato, COA 7340. Belalcázar a Santa Eufemia, Finca Picarazas, 1V-1978, M.L. Díaz, SEV 119955. Vega del Guadalquivir: Posadas a Palma del Río, Km 9, 12-V-1982, A. Pujadas, COA 7343. Hornachuelos-Palma del Río, 8-VI-1983, $A$. Pujadas, COA 7349. Cerca de Almodóvar del Río, 15-V-1987, J. Oliveira, COA 13652. Córdoba, Pgno. industrial del Sector Sur, 5-V1987, J. Oliveira, COA 13658. Almodóvar del Río, Finca Fuen-Real, 27-IV-1985, R. Soria \& J.M. Cabrera, COA 13659. Córdoba, INIA, 16III-1990, A. Pujadas, COA 13693. Valle del Guadalquivir: Bujalance a Córdoba, Km 2, margen izq. del Guadalquivir, 19-V-1982, A. Pujadas, COA 13862. Santaella, Arroyo Término,
17-V-1983, A. Pujadas, COA 7360. Puente Genil, Ribera baja, 26-III-1988, L. Velasco, COA 13859. El Carpio, 3-V-1988, J. Oliveira, COA 13699. Cerca de Montilla, 9-V-1989, A. Pujadas, COA 13506. Subbética Occidental: Cabra, Cerro de Camarena, 6-VI-1987, C. Gálvez, COA 13822. Priego de Córdoba., 22-VII-1987, J. Oliveira, COA 13774. Carcabuey, Pico Bermejo, 9-VI1989, A. Pujadas \& C. Gálvez, COA 13507. Base La Tiñosa, 15-V-1991, A. Lora \& A. Pujadas, COA 17521. Iznájar, cerro del Azucarón, 30SUG8526, 450 m, 2-V-1998, A. Pujadas, COA 25298. Luque, Laguna del Salobral, 18-V-2003, L. Plaza \& A. Dorda, COA 37572. GRANADA: Subbética Occidental: Sierra Parapanda, 15-III1990, E. Bullejos, COA 17523. Sierra Elvira, 25V-1991, P. Soto, COA 17524. Moclín, Cerrillo de la Solana, 27-IV-1996, J.F. Arcos, COA 22076. Carretera de Illora a Montefrío, VG 2128, 900 m, 21-IV-1983, Aroza et al., GDA 16814. Montillana, cortijo de las Juntas, 30SVG4147, 1120 m, 23-V-1970, C. Fernández López et al., JAEN 700163 (sub O. reticulata). Sierra de Mágina: Iznalloz, puerto de Onitar, 30SVG4744, 1060 m, 30-V-2000, C. Fernández López et al., JAEN 700204. Guadahortuna, cerro Atalaya, 30SVG6150, 1160 m, 3-VI-2000, E. Morales, JAEN 700991. Iznalloz, cortijo del Diablo, río Cubil, 30SVG5236, $760 \mathrm{~m}, 1-\mathrm{VI}-2001, C$. Fernández López et al., JAEN 610356. Serranía de Ronda: Sierra de Loja, Cerro de Las Monjas, 12- V-1989, J. Sánchez, COA 13823. Depresión de Granada: Silla del Moro, cerca del Aljibe de la lluvia, -1907-, J.L. Díez Tortosa, GDA 36793. Cogollos Vega, Las Horconas, 30SVG5327, 1350 m, 22-V-1988, O. Socorro et al., GDA 22368. Viznar, alrededores, 13-V-1975, T. Palomeque, JAEN 630653. Ventas de Huelma a Agrón, 1060 m, 24-VI-1975, T. Palomeque, JAEN 630632. Quéntar, alrededores, 9-V-1978, T. Palomeque, JAEN 630652. Sierras de Almijara, Guájares y Trevenque: Padul, 1-V-1987, J.J. Lara, COA 13654. Viznar a Alfacar, $1070 \mathrm{~m}, 13-\mathrm{VI}-1978, T$. Palomeque, JAEN 630650. Izbor, proximidades de la Venta de las Angustias, VF 5386, $550 \mathrm{~m}$, 26-III-1978, J. Molero Mesa, GDA 11252 (sub O. caryophyllaceae Sm. en Molero Mesa \& Pérez Raya, 1987: 266). Depresión de Guadix-Baza y Guadiana Menor: Cúllar a Galera, km 14, 30SWG3974, 920 m, 2-VI-2004, C. Fernández 
et al., JAEN 640696. Alpujarras bajas, Contraviesa y Sierra de Gádor: Motril, 24-III1970, Fernández Casas, MA 412237. Entre Salobreña y Almuñécar, 26-III-1977, E. Ruiz de Clavijo et al., SEV 162873. Sierra Nevada y Filabres: Trevélez, cerca del río Trevélez, 30-VI1990, A. Pujadas \& P. Poyato, COA 13583. Laroles, $1150 \mathrm{~m}$, 9-VI-1989, A. Hervás, GDAC 34734. Pampaneira, carretera de la Mina de la Virgen de Fátima, 7-IV-1978, Díaz et al., COFC 13036. Sierra de Baza: Baza, Fuentes de San Juan, 13-IV-1933, S. Rivas Goday, MAF 86256. HUELVA: Sierra de Aracena: Aracena, 22-VI1942, C.Vicioso, MA 114898 (Holotypus para O. clementei). Aracena, cordillera, 25-IV-1978, J. Rivera, SEV 48493. Andévalo: Huerto de las Palomas, El Granado, 29SPB3352, 18-VI-2001, E. Sánchez Gullón, COA 33985. Campiña de Huelva y Condado-Aljarafe: Camino desde Dehesa de Bollullos a Dehesa de Garruchena, 24IV-1994, S. Guitar \& S. Arias, COA 17475. Litoral: Cartaya, 5-IV-1987, J. Oliveira, COA 13730. Lepe, 29SPB6225, 9-IV-2002, E. Sánchez Gullón \& P. Weickert, COA 37568. Huelva, jardín, 29SPB8328, 15-IV-2003, E. Sánchez Gullón \& P. Weickert, COA 37570 . El Conquero, 29SPB8227, 25-IV-2003, E. Sánchez Gullón \& P. Weickert, COA 37566. Almonte, Doñana, Matalascañas, 28-V-1980, S. Castroviejo \& G. López, MA 435720. JAÉN: Molino Olmedo et al. (1995) aportan numerosas citas a las que añadimos las siguientes. Sierra Morena: Frailes, 14-IV-1988, J. Oliveira, COA 13515. Sierra de Andújar, 413212 / 4214421, 27-IV-2004, L. Plaza \& Ch. Velasco, COA 37520. Baños de la Encina, E1 Tesoro, 30SVH3527, 400 m, 10-VI-1984, J. Muñoz-Cobo, JAEN 841011. Guarromán a $1 \mathrm{~km}$ desde Linares, 30SVH4026, 9-VI-1993, C.Fernández \& S. Peñafiel, JAEN 931595 (sub O. amethystea en Molino Olmedo et al., 1993: 47). Valle del Guadalquivir: Fuensanta de Martos, 23-V-1996, A. Lora \& A. Pujadas, COA 22063. Jaén, Colegio Universitario, 30SVG3183, 340 m, 17-V-1984, M.A. Espinosa, JAEN 912924 (sub O. amethystea en Molino Olmedo et al., 1993: 47). Porcuna, 30SUG9692, 460 m, 10-VII-1990, J. Barranco \& J. Bellido, JAEN 900607 (sub O. amethystea en Molino Olmedo et al., 1993: 47). Baños de la Encina, Navarredondo y Chopera, 30SVH3024, 320 m, 28-IV-1991, JAEN 911112 (sub O. amethystea en Molino Olmedo et al., 1993: 47). Jaén, carretera de Granada, Jardín Los Olivos, 30SVG3880, $500 \mathrm{~m}, 15-\mathrm{V}-1993, C$. Fernández López, JAEN 930799 (sub O. amethystea en Molino Olmedo et al., 1993: 47). Torredelcampo Arroyo de Mingo López, 30SVG1589, 320 m, 16-V-1993, C. Fernández et al., JAEN 930854 (sub O. amethystea en Molino Olmedo et al., 1993: 47). Subbética Occidental: Puerto de la Sierra Pandera, $1600 \mathrm{~m}$, 12-VI-1991, A. Pujadas \& A. Lora, COA 17509. Cañones de Puente de la Sierra, 19-V-1992, $A$. Pujadas \& A. Lora, COA 17520. Sierra de Jabalcuz, 9-VI-1993, A. Pujadas et al., COA 17529. Jamilena a Martos, km 3, 21-V-2006, $A$. Pujadas \& E. Triano, COA 37634. La Guardia de Jaén, c. cerro de Torre Bermeja, 30SVG4081, 540 m, 27-IV-1991, A. González, JAEN 910552 (sub O. amethystea en Molino Olmedo et al., 1993: 47). Sierra de Mágina: Campanil, ad ripas fl. Bedmar, 500 m, 8-VI-1926, Cuatrecasas, BC 46656. Bedmar a Torreón de Cuadros, 30SVG6484, $600 \mathrm{~m}, 15-\mathrm{VI}-1995$, A. Pujadas \& A. Lora, COA 17662. Carchelejo, Ermita Santa Lucía, 23-V-1996, A. Pujadas \& A. Lora, COA 22065. Garcíez, hacia la estación de GarcíezJimena, 30SVG5993, 400 m, 11-V-1996, M.A. Espinosa et al., JAEN 960306. Versus Cambil, 800 m, 13-VI-1926, Cuatrecasas, MAF 36169. Cazorla-Segura-Las Villas: Cazorla, zona de las Monjas, 12-IV-1992, J.A. Rodero, COA 17626. Bruñel-Quesada, 9-V-1997, P. Luque, COA 23744. La Iruela, Juntas del Murel, 30SVH9803, 560 m, 18-V-1994, J. Fernández Liencres, JAEN 944928 \& JAEN 945489. Santiago de la Espada, prados en la cola del Embalse del Tranco, 30SVH1411, 640 m, 11-V-1980, C. Soriano, MA 457064. Depresión de Guadix-Baza y Guadiana Menor: Huesa, VG97, 500 m, 23-V-1979, C. Fernández López, JAEN 79683. MÁLAGA: Sierra del Aljibe: Gaucín, ctra. a Jimena, límite provincial, 30S0285709 / 4039406, $87 \mathrm{~m}, 13-\mathrm{V}-$ 2007, A. Pujadas, COA 37652. Castelar, 10-IV1976, T. Palomeque, JAEN 630664. Benarrabá, Los Pepes, 30STF9744, 150 m, 25-IV-2004, O. Gavira, MGC 61189. Algatocín, hacia Benalauría, 30STF9650, $800 \mathrm{~m}, 30-\mathrm{V}-2004, O$. Gavira, MGC 61190. Playas de Casares, 28-IV1978, T. Luque et al., SEV 162882. Serranía de Ronda: Sierra de Torremolinos, 29-IV-1984, S. 
Pérez Sanz, MGC 16535. Sierra Blanca, cercanías a Ojén, 7-V-1982, Merino, MGC 11780. Alhaurín de la Torre, Sierra de Mijas, Los Caracolillos, 27IV-1984, M. Lerga \& A. Villadres, MGC 16534. Puerto Cabo Pino, Marbella, 22-III-1985, G. Michel, MGC 21242. Benaoján, carretera hacia Ronda, 30SUF0067, 460 m, 23-IV-1988, D. Montilla, MGC 40474. Axarquía: Casabermeja, 12-V-1988, J. Oliveira, COA 13782. Colmenar, 15-VI-1992, J.A. Gómez, COA 22075. Guaro, 24VI-1992, A. Pujadas et al., COA 13584. Nerja, c. Cuevas de Nerja, en Maro, 17-IV-1984, J.L. Fernández Alonso et al., MA 519947. Rincón de La Victoria, UF 8164, 27-IV-1984, P. Sáenz \& Hidalgo, MGC 14009. Salida de Periana, 13-IV1989, B. Cabezudo et al., MGC 29645. Depresión de Antequera: Antequera, a $15 \mathrm{~km}, 12-\mathrm{V}-1988$, J. Oliveira, COA 13704. Cerca de Antequera, 28IV-1988, J. Oliveira, COA 13791. Sierra Yeguas, Cortijo Peñuelas, 11-VI-1996, M.A. Díaz, COA 19845. Salida de Antequera hacia Campillos, $17-$ V-1989, $M^{a}$ M. Trigo, MGC 29644. Sierra de Almijara, Guájares y Trevenque: Sierra Tejeda, subida al Pico Maroma, 25-V-1991, A. Pujadas et al., COA 17510. Alcucín, Sierra Tejeda, entre Alcaucín y el puente Don Manuel, 19-V-1982, J.M. Nieto \& B. Cabezudo, MGC 41506. SEVILLA: Sierra Morena: Las Navas de la Concepción, Guadalora, 7-V-1989, A. Vargas, COA 13779. Entre el cruce del embalse del Río Retortillo y Puebla de los Infantes, 6-V-1978, M. López et al., SEV 120069. Desviación de la carretera de Lora del Río a Constantina, 7-V1978, M. López et al., SEV 119971. Constantina, 3-VI-1981, P. Escalza et al., SEV 120723. Campiña de Huelva y Condado-Aljarafe: Aznalcázar, 5-IV-1987, J. Álvarez, COA 13574. Entre Castilleja del Campo y Sanlúcar la Mayor, 20-VI-1978, S. Talavera \& B. Valdés, SEV 162915. Cuesta de las Doblas, 19-V-1984, D. Cabezas et al., SEV 105375. Vega del Guadalquivir: Brenes, 5-V-1984, A. Lara, COA 13656. Vega de Lora del Río, 15-V-1989, E. Gómez, COA 13858. Sevilla, jardín de la Universidad, 26-III-1968, S. Silvestre \& B. Valdés, SEV 110045. Coria del Río, 6-IV-1977, S. Silvestre, SEV 27875. Villaverde del Río, 6V-1983, I. Redondo et al., SEV 119972. Litoral: Carretera Aznalcázar-Isla Mayor, 4-III-1977, F. Garcia, SEV 162920. Valle del Guadalquivir:
Mairena, 15-V-1984, A. Borrero, COA 13655. Utrera, 6-IV-1988, J. Oliveira, COA 13670. Ecija a Herrera, Km 25, 5-IV-1988, J. Oliveira, COA 13674. Lebrija a El Cuervo, Km 4, 6-IV-1988, J. Oliveira, COA 13683. Osuna a Estepa, Km 10, 5-IV-1987, A. Pujadas, COA 13684. El Palmar, 6-IV-1988, J. Oliveira, COA 13700. Cabezas de San Juan, 6-IV-1988, J. Oliveira, COA 13731.

Como ya indicara Valdés (1987: 351) es muy frecuente en Valle del Guadalquivir pero también en Subbética Occidental y Serranía de Ronda de donde el mismo autor indica la presencia de $O$. reticulata, probablemente, por confusión con $O$. crenata puesto que muchos de los pliegos identificados bajo ese nombre corresponden a $O$. crenata.

22. O. amethystea Thuill., Fl. Env. Paris, ed. 2, 317 (1800)

- Brácteas que superan la longitud de las flores. Dientes de cáliz estrechos, ápice subfiliforme, igualando la longitud del tubo de la corola. Corola marcadamente inflexa por encima de la inserción de los filamentos. Labio superior de la corola emarginado. Estigma morado ... $\alpha$. subsp. amethystea

- Brácteas que igualan la longitud de las flores. Dientes del cáliz anchos o estrechos, ápice lanceolado o subfiliforme, menores o igualando la longitud del tubo de la corola. Corola ligeramente inflexa por encima de la inserción de los filamentos Labio superior de la corola íntegro o emarginado. Estigma amarillo, anaranjado o morado

$\boldsymbol{\beta}$. subsp. castellana

\section{$\alpha$. subsp. amethystea}

Presente en Alpujarras bajas, Contraviesa y Sierra de Gádor, Sierra Nevada y Filabres, Sierra de María, Orce y Estancias, Altiplanos Manchegos y Almería Subbética, Almería Subdesértica (Pujadas \& Lora, 1995: 52; Pujadas Salvà \& Lora González, 1996: 213-214). Aportamos nuevas citas que amplían y confirman su área de distribución. (UICN: NT).

ALMERÍA: Alpujarras bajas, Contraviesa y Sierra de Gádor: Fuente Cufría, 17-V-1974, $R$. Sagredo, $\mathrm{H}^{\mathrm{a}}$ La Salle ALMERÍA: Sierra de Gádor, Fuente Victoria, Cerro Alto, WF0990, B. Valdés et al., SEV s/n. Sierra Nevada y Filabres: Benizalón, Cortijo Pajares, 30SWG6617, $850 \mathrm{~m}$, 30-IV-1988, C. Morales \& A.B. Robles, GDA 48843. Sierra de María, Orce y Estancias: 
Barranco Mula, WG6265, 1500 m, 8-VI-1988, $M$. Cueto, ALME 3039 (sub O. minor en Pujadas Salvà \& Lora González, 1996: 215). Subida al Maimón, 30SWG7871, 1260 m, 23-VI-1976, Castroviejo \& Valdés-Bermejo, MA 435506. Almería Subdesértica: Adra, La Parra, El Romeral, 10-V-2002, 30SVF9469, J. Peñas, HUAL 7258. Sur de Mojácar, urbanización Lomas Cantal, 30-V-1971, Fernández Casas, MA 412238. Sierra Alhamilla, Peñón de Turrillas, WF69, 900-1000 m, 7-VI-1967, P.W. Ball et al., SEV 4864. CÁDIZ: Litoral: Vejer de la Frontera, Finca La Muela, 15-IV-1988, E. Morillo, COA 13529. Laguna Campano, Chiclana, 22-IV-1990, A. Pujadas et al., COA 17610. Entre Chipiona y Rota, 19-IV-1978, J.A. Devesa \& J. Pastor, SEV162955. Valle del Guadalquivir: Jerez de la Frontera, 11-V-1988, A. Pujadas \& J. Oliveira, COA 13539. Sierra del Aljibe. Algeciras, Los Alcornocales, Arroyo La Miel, c. Cobre, 19-V2001, L. Plaza \& J. Salcedo, COA 34006. Los Barrios, Sierra de la Palma, 22-IV-1980, J. Arroyo \& J.M. Gil, SEV 71581. Tarifa, Sierra del Niño, Arroyo Bugones, 10-V-1980, J. Arroyo et al., SEV 71681. Los Barrios, Sierra del Niño, Arroyo del Tiradero, casa de la Angarilla, 10-V-1980, J. Arroyo \& S. Silvestre, SEV 71682. Los Barrios, Sierra de Luna, entre la Alcaria y las Gargantillas, 11-V-1980, J. Arroyo et al., SEV 71822. Serranía de Ronda: Benaocaz, subida a casa Fardela, 16VII-1998, L. Plaza et al., COA 34008. P.N. Grazalema, Arroyo del Alcornocalejo, 12-V2002, C. Burgarella \& L. Plaza, COA 34012. Algodonales, Sierra de Líjar, 31-V-1980, A. Aparicio, SEV 115462. El Gastor, Sierra del Gastor, TF9280, 29-V-1983, A. Aparicio et al., SEV 115466. Villaluenga del Rosario, Sierra del Caillo, Navazo Alto, TF8564, 30-VI-1983, A. Aparicio, SEV 11569. CÓRDOBA: Sierra Morena: Obejo, 18-V-2001, A. Pujadas \& D. Rubiales, COA 34002. Hornachuelos, Centro Interpretación Naturaleza, 28-IV-2003, A. Moral, COA 37576. Espiel, Sierra de Espiel, 30S0321601 / 4226378, $590 \mathrm{~m}, 8-\mathrm{V}-2007, A$. Pujadas, COA 37645. Hornachuelos, de Hornachuelos a Posadas, 305350 / 4188841, 128 m, 14-V-2007, E. Reyes Sepúlveda, COA. Subbética Occidental: Ermita de Cabra, 22-V1991, A. Pujadas \& M. Gisbert, COA 17607. Camino de Zuheros a la Nava, 25-V-1996, M.L.
Sillero, COA 23738. Rute, base Sierra Alta, 30SVG8134, 29-VI-1996, A. Pujadas \& A. Lora, COA 30472. Carcabuey, Los Pollos, 20-VI-2000, N. Molina, COA 37515. Luque, c. Laguna del Salobral, 18-V-2003, L. Plaza \& A. Dorda, COA 37535. GRANADA: Subbética Occidental: Algarinejo, c. Cortijo Bujeo Alto, 30SUG9130, 675 m, 15-V-1999, E. Triano \& R. Hinojosa, COA 29279. Montefrío, 4-VI-2000, D. Rubiales, COA 29891. Sierra de Parapanda, Illora, 30SVG1831, 1400, 7-VI-2000, A. Pujadas, COA 29892. Cazorla-Segura-Las Villas: Huéscar, Sierra de Guillimona, 30SWH4009, 1750 m, 6-VI-2002, C. Morales \& G. Blanca, GDA 46266. Serranía de Ronda: Loja, Sierra de Loja, Base cerro Las Monjas, 30SVG9812, 800 m, 12-V-1989, M.U. Garcia \& M.J. Salinas, GDA 33682. Sierras de Almijara, Guájares y Trevenque: Alhama de Granada, 30SVF1096, 16-VI-1991, A. Pujadas \& P. Poyato, COA 16485. Sierra Tejeda, Loma de las Víboras, VF0487, 27-V-1984, J.M. Nieto \& B. Cabezudo, MGC 41505. Alhama de Granada, P.N. Sierra Tejeda, Almijara y Alhama, Barranco del Malinfierno, 30SVF1585, 1200-1300 m, 17VI-2004, B. Cabezudo et al., MGC 59652. Depresión de Guadix-Baza y Guadiana Menor: Guadix, 5-IV-1997, J. Pusch, COA 23581. CúllarBaza, Km 2 hacia Huéscar, 3-V-2000, A. Pujadas \& J.A. López, COA 29896. Zújar, carril del Jabalcón, 30SWG1757, 1020 m, 28-V-2004, C. Fernández et al., JAEN 640584. Alpujarras bajas, Contraviesa y Sierra de Gádor: Alpujarras, El Colco, 1-VII-1990, A. Pujadas \& P. Poyato, COA 13604. Sierra Nevada y Filabres: Cerca del cortijo Calvache, Trevélez., 16-VII-1990, A. Pujadas \& P. Poyato, COA 17605. Huéneja, umbría Sierra Nevada, 1700 m, 21-VI-1994, A. Pujadas \& A. Pallarés, COA 16486. Sierra Nevada, 1888, $C$. Voig, COI-H ${ }^{\circ}$ Willkomm. Carril subida Puerto de la Ragua, 1650 m, 10-VI-1997, M. Salinas et al., GDAC 43957. Sierra de Baza: Sierra de Baza, borde de un arroyo, $1500 \mathrm{~m}, 22$-VII-1975, B. Cabezudo \& S. Talavera, SEV 162937. HUELVA: Sierra de Aracena: Castaño del Robledo, pico Castaño, 30SOB0396, 7-V-1996, M. López, COA 24652. Cumbres de San Bartolomé, 30SPC9317, 22-IV-1998, M. López, COA 25301. Jabugo, 19-V-1988, M. López, COA 24659. Ribera del Jabugo, P.N. Aracena, 29SQC0097, VIII-2005, E. Sánchez Gullón \& P. 
Villegas, COA 3758. Linares de La Sierra, cordillera, $700 \mathrm{~m}, 15-\mathrm{V}-1979$, J. Rivera, SEV 48497. Campiña de Huelva y Condado-Aljarafe: Villarrasa, El Alcornocal, 29SQB1137, 18-IV2001, E. Sánchez Gullón, COA 34000. El Villar, Bonares, 29SQB0522, E. Sánchez Gullón, COA 34001. JAÉN: Molino Olmedo et al. (1995: 47) aportan abundantes citas a las que añadimos las siguientes. Sierra Morena: Linares a Baños, 6VI-1992, A. del Campo, COA 17631. De las Correderas a Santa Elena, 12-VI-1941, MAF 36186. Valle del Guadalquivir: De Martos a Lendinez, Km 8, 12-VI-1991, A. Pujadas \& A. Lora, COA 17513. Martos, Peña, VG1475, 21 V-1977, C. Fernández, JAEN 770037 (sub O.crenata en Molino Olmedo et al., 1995: 48). Subbética Occidental: Los Villares, subida al Puerto de la Pandera, 1600 m, 12-VI-1991, A. Pujadas \& A. Lora, COA 30474. Sierra de Jabalcuz, 9-VI-1992, A. Pujadas et al., COA 13496. Alcaudete, S. de Orbes, VG06, 720 m, 25V-1977, C. Fernández López, JAEN 771094 (sub O.minor en Molino Olmedo et al., 1995: 49). Sierra de Mágina: Campanil, ad ripas fl. Bedmar, 800 m, 8-VI-1926, Cuatrecasas, BC 46677. Almadén, mont. Cabezaprieta, $1500 \mathrm{~m}, 11-\mathrm{VI}-$ 1926, Cuatrecasas, BC 46681. Base Carluco, casa de la subida. Albánchez, 30SVG6282, $1000 \mathrm{~m}$, 15-VI-1995, A. Pujadas \& A. Lora, COA 17663. Torres a Albánchez, Km 2, 30SVG5681, 1000 m, 15-VI-1995, A. Pujadas \& A. Lora, COA 17664. Cabra del Sto. Cristo, La Silleta, 2-VII-1998, $A$. Pujadas \& A. Lora, COA 34011. Cazorla-SeguraLas Villas: Nacimiento R. Guadalquivir, 15-VIII1997, J. Madrigal \& L.F. Perea, COA 30475. Falda de Poyos de La Mesa, J. Madrigal \& L.F. Perea, COA 30476. Sierra Cazorla, crossroads towards Nacimiento, WH0497, 1100 m, 30-VI1988, B. Valdés et al., SEV. MÁLAGA: Sierra del Aljibe: Gaucín, 30S0291270 / 4043302, 644 m, 13-V-2007, A. Pujadas, COA 37654. Jubrique, Cerro Hoyones, 30SUF0249, 600 m, 5-VI-2004, O. Gavira, MGC 61188. Casares, subida a Casares, 28-IV-1978, T. Luque et al., SEV 162872 \& SEV 162876. Atajate, base del Conio, 30SUF0059, $1020 \mathrm{~m}, 28-\mathrm{V}-2006$, O. Gavira, MGC 64772. Serranía de Ronda: Sierra de los Cañetes, 17-VII-1991, A. Pujadas \& A. Lora, COA 17612. Valle de Abdalajís. Sierra del Valle de Abdalajís, El Capilla, 30SUF4690, 21-VI-
1997, L. Plaza et al., COA 23365. Antequera, Sierra Huma, Haza del Río, 30SUF4386, 23-IV1997, L. Plaza et al., COA 24141. Torcal de Antequera, 26-V-2000, B. Román, COA 29890. Istán, Alborque, UF2754, 4-V-1990, $M$. Hernández \& A. Navas, MGC 30434. Benaoján, Sierra del Palo, TF9461, 1250 m, 27-VI-2004, M. Becerra, MGC 59806. Axarquía: Riogordo, 20-V-1989, A. Pujadas, COA 13492. Periana, 20V-1989, A. Pujadas, COA 13497. Cortijo de Marchamonas, Periana, 30SUF9591, 900 m, 24VI-1992, A. Lora et al., COA 22223. Alhaurín de la Torre, 30SUF5757, 12-IV-1998, R.R. Platero et al., COA 27234. SEVILLA: Sierra Morena: Guadalcanal, Sierra del Viento, 9-VI-2001, L. Plaza \& J. Salcedo, COA 34009. San Nicolás del Puerto, Arroyo Húmedo, 22-VI-1976, E.F. Galiano et al., SEV 110039. Valle del Guadalquivir: Osuna, c. Cerro Cantalejo, 30SUG1515, 15-VI-2001, J. Salcedo, COA 34007. Carmona, Ermita de Santa María de Gracia, 24-IV-1979, P. Candau et al., SEV 162892.

B. subsp. castellana (Reut.) Rouy in Rouy \& Foucaud, Fl. France 11: 185 (1909)

Orobanche amethystea var. henriquesi J.A. Guim., Brotéria 3: 134 (1904)

CÓRDOBA: Alcaracejos, La Chimorra, 333309 / 4230727, 762 m, 15-V-2007, E. Reyes Sepúlveda, COA 37649. HUELVA: Sierra de Aracena: Linares de la Sierra a Aracena, Km 3, 30SQB1296, 7-V-1997, A. Pujadas, COA 23739. Aracena a Linares de la Sierra, Km 5, 17-V-2002, A. Pujadas, COA 33954.

Categoría UICN: CR [B2ac(iv)]

Observaciones. O. amethystea subsp. castellana, no ha sido citada por Valdés (1987) para Andalucía Occidental. No hemos podido encontrar otros testimonios fiables de este taxón. Aunque existen referencias bibliográficas sobre su presencia (Molino Olmedo et al., 1995: 47) creemos que se trata de errores de identificación.

23. O. almeriensis A. Pujadas, Anales Jard. Bot. Madrid 53(1): 48 (1995)

Orobanche amethystea var. almeriensis (A. Pujadas) A. Pujadas, Plantas Parásitas de la Península Ibérica e Islas Baleares 395 (2002) Presente en Sierras de María, Orce y 
Estancias (Pujadas \& Lora, 1995; Pujadas Salvà \& Lora González, 1996: 214). Aportamos nuevas citas que amplían su área de distribución.

ALMERÍA: Sierra de María, Orce y Estancias: Sierra de María, c. Ermita Virgen de la Cabeza, WG7172, 1420 m, 22-VI-1988, M. Cueto, ALME 14931 (sub O. crenata en Pujadas Salvà \& Lora González, 1996: 213). Albox, Santuario El Saliente, 30SWG7453, 900 m, 27IV-2002, A. Pujadas et al., COA 33987. De Vélez Rubio al Puerto de Santa $\mathrm{M}^{\mathrm{a}}$ de Nieva, Km 6, 26IV-2002, A. Pujadas et al., COA 33986. Altiplanos Manchegos y Almería Subbética: Vélez Blanco, El Gabar, 30S0575487 / 41777409, 1066 m, 26-IV-2007, A. Pujadas \& A. Ivorra, COA 37660. Vélez Blanco, base Peña de los Letreros, 30S0579798 / 4170541, 1030 m, 26-IV2007, A. Pujadas \& A. Ivorra, COA 37665. Vélez Blanco, de El Gabar a Vélez Blanco, 30S0578448 / 4174018, 1030 m, 26-IV-2007, A. Pujadas \& A. Ivorra, COA. Almería Subdesértica: Alhabia, 30SWF3893, 300 m, 25-IV-1994, A. Pujadas, COA 16480 (sub O. amethystea en Pujadas \& Lora, 1995: 52). Cantoria-Albánchez, Km 3, 30SWG7443, 25-V-1994, A. Pujadas, COA 16474 (sub O. amethystea en Pujadas \& Lora, 1995: 52). Barranco de Santa Fe de Mondújar, 30SWF4294, 250 m, 27-V-1994, A. Pujadas, COA 16470 (sub O. amethystea en Pujadas \& Lora, 1995: 52). GRANADA: Depresión de Guadix-Baza y Guadiana Menor: Benamaurel, c. Cuevas Luna, 30S0524689 / 4162200, 685 m, 28IV-2006, A. Pujadas \& L. Plaza, COA 37519. Benamaurel, Castillejar, yesos y río, 30SWG3173, 750 m, 5-VI-2004, C. Fernández et al., JAEN 640783. JAÉN: Subbética Occidental: Los Villares, Río Frío, VG36, 1250 m, 14-VI-1977, C. Fernández López, JAEN 771579 (sub O. crenata en Molino Olmedo et al., 1995: 48).

\section{Categoría UICN: VU [B1a; D1]}

Observaciones. Foley (2001b: 57) la incluye provisionalmente en $O$. calendulae Pomel.

24. O. santolinae Loscos \& J. Pardo in Willk. (ed.), Ser. Inconf. Pl. Aragon.: 79 (1863)

Orobanche artemisiae-campestris subsp. santolinae (Loscos \& J. Pardo) O. de Bolòs, Vigo, R.M. Masalles \& J.M. Ninot, Fl. Man. Països Catalans 1214 (1990)
O. picridis sensu auct. non F.W. Schultz, Bot. Lit.-Blätt. 5 : 504 (1830)

ALMERÍA: Almería Subdesértica: Tabernas, km 10 al Este de Tabernas, Venta de los Yesos, WG60, 520-530 m, gypsum hills, 3VI-1967, P.W. Ball et al., ALME 1709 \& SEV 4865 (sub O. minor en Pujadas Salvà \& Lora González, 1996: 215). Venta de los Yesos, entre Tabernas y Sorbas, 30SWG60, 500-550 m, 13V-1981, R. Lázaro Suau, ALME \& MGC 13089. (sub O. minor en Pujadas Salvà \& Lora González, 1996: 215). Tabernas, Venta de los Yesos, 30SWG6204, 18-V-1996, A. Pujadas, COA 23563. Venta de los Yesos, a $2 \mathrm{~km}$ de Uleila, 19IV-1967, R. Sagredo, $\mathrm{H}^{\circ}$ La Salle Almería. Tabernas, Venta de los Yesos, 30SWG6203, 560 m, 25-IV-1998, F.J. Pérez-Garcia, HUAL 6914. CÓRDOBA: Subbética Occidental: Sierra de Rute, 30SUG8035, $800 \mathrm{~m}, 11-\mathrm{V}-1997, A$. Pujadas, COA 23560. Carcabuey, El Palancar, 16-V-2004, E. Triano \& A. Pujadas, COA 37632. GRANADA: Subbética Occidental: Sierra de Parapanda, 30SVG1931, 1200 m, 7-VI-2000, $A$. Pujadas \& E. Triano, COA 32136. Sierra de Madrid, Lagunazo, VG2230, 1200 m, 25-VI1982, Aroza Montes et al., GDA 1558. Sierra Mágina: Ventas de Huelma a Cacín, cerca de Odricha, 27-V-1999, D. Rubiales, COA 29307. Domingo Pérez, Cotifar, 30SVG5653, $1000 \mathrm{~m}$, 25-V-2002, C. Fernández et al., JAEN 620250. Dehesas Viejas a Cortijo Cañada Hermosa, 30SVG5347, 970 m, 18-V-2002, C. Fernández et al., JAEN 620209. Sierras de Almijara, Guájares y Trevenque: Trevenque, 6-IX-1991, $A$. Pujadas et al., COA 16489. Base del Trevenque, 1700 m, 12-VII-1996, A. Pujadas \& A. Lora, COA 24870. Puerto de la Mora, 1200 m, 13-VI1979, T. Palomeque, JAEN 630640. JAÉN: Valle del Guadalquivir: La Carolina, La Aliseda, VH44, 650 m, 2-VI-1979, C. Fernández López, JAEN 79879 (sub O.minor en Molino Olmedo et al., 1995: 49). Subbética Occidental: Castillo de Locubín a Valdepeñas de Jaén, a $3 \mathrm{Km}$ desviación al nacimiento Río San Juan, 23-V-1996, A. Pujadas \& A. Lora, COA 29459. MÁLAGA: Ronda: Antequera, cara norte de Sierra de Huma, 30SUF4488, 6-VI-1997, L. Plaza et al., COA 24142.

Categoría UICN: NT.

Observaciones. 
No citada por Valdés (1987) para Andalucía Occidental. Con frecuencia identificada como $O$. minor o como $O$. picridis. Identificada por nosotros como $O$. minor en Pujadas Salvà \& Lora González (1996: 215) para Almería. Foley (2001b: 58) la incluye erróneamente en $O$. artemisiae-campestris Vaucher ex Gaudin.

25. O. minor Sm. in Sowerby, Engl. Bot. 6: tab. 422 (1797)

Orobanche barbata Poir. in Lam., Encycl. $4: 621$ (1798) p.p.

Presente en Sierra Morena, Sierra del Aljibe, Sierras de María, Orce y Estancias y Almería Subdesértica (Pujadas Salvà \& Lora González, 1996: 215; Pujadas Salvà, 1997: 34). Aportamos nuevas citas que amplían o confirman su área de distribución.

ALMERÍA: Sierras de María, Orce y Estancias: Oria, Los Cerritos, arroyo del Saliente, 30SWG7254, $900 \mathrm{~m}, 11-\mathrm{V}-1994$, A. Pujadas, COA 17442. El Saliente, 900 m, 19-V-2006, A. Pujadas \& B. Pérez-Vich, COA 37574. Altiplanos Manchegos y Almería Subbética: Vélez Blanco, El Gabar, 30S0578400 / 4178375, 986 m, 26-IV2007, A. Pujadas \& A. Ivorra, COA 37663. Almería Subdesértica: Playa del Pozo del Esparto, 30SXG1633, 14-IV-2005, A. Pujadas, COA 33982. Carboneras, 20 m, 27-VI-1976, $T$. Palomeque, JAEN 630695. CÁDIZ: Litoral: Chiclana de La Frontera, Pinar del Hierro, 29SQA6030, 23-V-1998, L. Plaza \& M.L. Sillero, COA 37596. Barbate, pinar costero, 15-VI-2000, E. Sánchez Gullón, COA 29590. Los Majales del Sol, Pinar de la Breña, Barbate, -V-2002, E. Sánchez Gullón \& P. Weickert, COA 37595. Puerto Real, Caño Río San Pedro, 3-V-2003, D. Rubiales, COA 37594. Puerto de Santa María, 19V-1873, M. Winkler, COI-H ${ }^{\circ}$ Willkomm (sub $O$. barbata Poir.; citado en Willkomm, 1893: 189, como O. minor Sutt.). Lapiedra, près Puerto de Santa María, 3-V-1849, E. Bourgeau, MANCH 370262. Sierra del Aljibe: Parque de los Alcornocales, Benalup, Finca El Zapatero, 30STF6023, 300 m, 4-V-1995, A. Pujadas \& A. Lora, COA 17620. P.N. Los Alcornocales, Sierra del Niño, base cerro Carboneros, 30STE6807, 20VI-1995, A. Lora et al., COA 37619. Facinas, km 6 de Facinas a Los Barrios, 10-V-2003, A. Pujadas, COA 37604. Los Barrios, 10-V-2003,
A. Pujadas, COA 37603. Algeciras, Río de La Miel, 24-V-2004, A. Pujadas et al., COA 37597. Algeciras, 27-VI-1887, E. Reverchon, MANCH 370069. Serranía de Ronda: P.N. de Grazalema, $\mathrm{S}^{\mathrm{a}}$ del Endrinal, 30STF8769, 21-VI-1995, A. Lora et al., COA 37623. Zahara, Garganta de Bocaleones, TF 8577, 13-V-1983, A. Aparicio et al., SEV 115468. Algodonales, Sierra de Algodonales, TF8484, $600 \mathrm{~m}, 30-\mathrm{IV}-1984, A$. Aparicio \& J.G. Rowe, SEV 115464. Sierra de Grazalema, entre el Puerto del Boyar y el Pozo de las Presillas, 1200 m, 29-V-1990, A. Aparicio \& S. Silvestre, SEV 162909. El Bosque, Km 1718 a Prado del Rey, TF7572, 20-V-1983, A. Aparicio \& J. Blázquez, SEV. CÓRDOBA: Sierra Morena: De Montoro a Cardeña, km 9, 24-V1983, A. Pujadas, COA 7341. Cerca de Villaviciosa, 4-VI-1989, A. Pujadas \& P. Poyato, COA 13517. De Obejo a Villanueva de Córdoba, 26-IV-1983, A. Pujadas, COA 13519. Córdoba a Villaviciosa, cerca de Los Arenales, 1-X-1985, A. Pujadas \& P. Poyato, COA 13542. Córdoba, Arroyo Bejarano, 21-IV-2004, Ch. Velasco \& Ú. Osuna, COA 37609. Río Guadiato, Mesas Altas y Cerro del Caballo, 3-V-1979, Varela, COFC 1822 \& MA 294116 (sub O. alba en Foley, 2001b: 52). Pedroches: Arroyo Corcobada, Km 5 al Sur de Cardeña, 30SUH8631, 720 m, 24-V-1989, $A$. Pujadas \& J. Oliveira, COA 13494. Cardeña, Sendero de la Vegueta, 23-IV-2006, L. Plaza et al., COA 37626. Vega del Guadalquivir: Posadas a Palma del Río, km 33-34, 17-IV-1998, L. Plaza, COA 37636. Carretera Córdoba a Sevilla, arroyo que desemboca en el Guadalquivir a la altura del P. sifón, sector XII, 18-IV-1980, Fernández \& Porras, COFC 1498. Valle del Guadalquivir: Puente Genil, 24-V-1989, J. Oliveira, COA 13871. Subbética Occidental: Carcabuey, castillo, 29-IV-2002, E. Triano, COA 37644 (fotografía). GIBRALTAR: Sierra del Aljibe: Gibraltar, between Sprague Farm and San Roque, 12-V-53, Wolley-Dod, PRC (sub O. mauritanica Beck). GRANADA: Cazorla-Segura-Las Villas: Huéscar, Sierra de Moncayo, WG3395, 22-VI1988, B. Valdés et al., Itinera 1650, SEV s/n (en Valdés \& Talavera, 1991: 204). Sierra de Guillimona, gorge of Torilla, WH3812, 1400$1500 \mathrm{~m}, 23-\mathrm{VI}-1988$, B. Valdés et al., Itinera 1687, SEV s/n (en Valdés \& Talavera, 1991: 204). Depresión de Granada: Entrada a Santa Cruz del 
Comercio desde Alhama de Granada, s/f, $C$. Burgarella, COA 37633. Sierras de Almijara, Guájares y Trevenque: La Zubia, cortijo Corvales, 1000 m, 3-V-2002, C. Burgarella \& L. Plaza, COA 37577. Cogollos Vega, camino a Cortijo de Carialfaquín, 30SVG5225, 1100 m, 14-IV-1988, L. Cano, GDA 22363. La Caleta, VF46, 28-III1978, R.M. Burton, SEV 35552. HUELVA: Sierra de Aracena: Alájar, Los Madroñeros, 10-IV-1998, L. Plaza, COA 37610. Aracena a Linares de la Sierra, km 6, 30SQB1296, 17-V-2002, A. Pujadas, COA 30528. Encima de Linares de la Sierra, 7-IV-1978, J. Rivera, SEV 48495. Andévalo: El Granado, Huerta de las Palomas, 18-VI-2001, E. Sánchez Gullón. COA 33985. Campiña de Huelva y Condado-Aljarafe: Aljaraque, Los Medios, 29SPB7528, 25-IV-2003, E. Sánchez Gullón \& P. Weickert, COA 37625. Litoral: Cartaya, El Rompido, 30SPB6820, 23 II-2000, E. Sánchez Gullón, COA 29592. Marismas del Odiel, 29SPB8514, 14-IV-2000, E. Sánchez Gullón, COA 29581. Isla Cristina, 15III-2002, E. Sánchez Gullón, COA 37581. Ayamonte, Punta del Moral, 29SPB4515, IV2002, E. Sánchez Gullón \& P. Weickert, COA 37599. Cartaya, Lagunas del Lancón, 29SPB6421, IV-2002, E. Sánchez Gullón \& P. Weickert, COA 37600. Ayamonte, Isla Canela, 1V-2005, A. Pujadas \& P. Pujadas, COA 37536. Huelva, Jardín Botánico Dunas del Odiel, 19-IV2006, L. Plaza, COA 37598. JAÉN: Citas de Molino Olmedo et al. (1995: 49), además aportamos las siguientes referencias. Sierra Morena: Aldeaquemada, camino de la Cimbarra, 27-V-1953, Galiano, SEV 4862. Valle del Guadalquivir: Baeza, Castillo del Jarafe, colina cercana, 30SVG5094, $500 \mathrm{~m}, 14-\mathrm{V}-1993, C$. Fernández et al., JAEN 931239. Vilches, La Casería, 30SVH5331, 520 m, 22-V-1996, J.L. Hervás, JAEN 963044. Subbética Occidental: Sierra de Jabalcuz, Los Cañones Secos, 1-VI2000, B. Román \& F. Fiestas, COA 29926. Fuente de la Peña, 30SVG2979, 600 m, 20-V-1983, $T$. Palomeque, JAEN 832436. Sierra Mágina: Entre Cambil y Huelma, 2-VII-1998, L. Plaza \& M.L. Sillero, COA 37576. Carboneras, 1300 m, 3-VII1925, Cuatrecasas, BC 46693. MÁLAGA: Sierra del Aljibe: Benarrabá, Los Pepes, 30STF9744, 150 m, 25-IV-2004, O. Gavira, MGC 57714. Serranía de Ronda: Marchamonas, 30SUF9591,
900 m, 4-VI-1992, A. Lora et al., COA 17619. Sierra Blanca, Marbella, La Alpujata, 3-V-2003, P. Blanco, COA 37575. Cartájima, prox. Cortijo de las Azuzaderas, 30SUF0759, $940 \mathrm{~m}, 8$-IV1989, D. Montilla, MGC. Benaoján, La Dehesa, 30STF9864, 500 m, 5-V-2002, M. Becerro, MGC 51774. Antequera, Sierra de la Chimenea, 15-VI1973, S. Talavera \& B. Valdés, SEV 119961. Axarquía: Alhaurín de la Torre, acceso Tiro Pichón, 30SUF5757, 12-IV-1998, R.R. Platero et al., COA 27244. Alhaurín de la Torre, subida a Coto Alarcón, 14-VI-1998, R. Rubio, COA 27239. Alhaurín de la Torre, c. Cortijo La Mezquita, 30SUF5555, 16-VI-1998, R.R. Platero et al., COA 27240. Sierra de Cártama, 21-IV-1973, 350 m, S. Talavera \& B. Valdés, SEV 119960. SEVILLA: Sierra Morena: Cazalla de la Sierra, Embalse del Pintado, 6-IV-2002, L. Plaza \& J. Salcedo, COA 37612. San Nicolás del Puerto, Cerro del Hierro, 29-IV-2004, Ch. Velasco \& J.M. Delgado, COA 37624. Valle del Guadalquivir: Dos Hermanas, 21-IV-1895, C. Pau, BC 46696 \& MA 114976. Osuna, cerro Cantalejo, UG1515, 15-VI-2001, J. Salcedo, COA 37613.

Categoría UICN: LC

Observaciones. Gran parte de las referencias de $O$. minor en Pujadas Salvà \& Lora González, (1996: 215) corresponden a O. ballotae (cf. Pujadas Salvà (1997) y a O. santolinae como se ha indicado anteriormente.

26. O. ballotae A. Pujadas, Acta Bot. Malacitana 22: 30 (1997)

Orobanche minor fma. albens sensu A. Pujadas \& A. Lora, non Beck, Corintia 2: 108 109 (1821)

O. minor var. palaestina sensu Pau, non $O$. palaestina Reut. in DC, Prodr. 11: 718 (1847)

Presente en Vega del Guadalquivir, Subbética Occidental, Axarquía, Sierras de Almijara, Guájares y Trevenque, Almería Subdesértica (Pujadas Salvà, 1997: 34). Aportamos nuevas citas que amplían su área de distribución.

ALMERÍA: Alpujarras bajas, Contraviesa y Sierra de Gádor: Alcolea, 6-V-1960, R. Sagredo, $\mathrm{H}^{\circ}$ La Salle Almería (sub O. minor en Pujadas \& Lora, 1996). Almería Subdesértica: Barranco del Sabinar, 16-IV-1962, R. Sagredo, $\mathrm{H}^{\circ}$ La Salle Almería (sub O. minor en Pujadas \& Lora, 1996). 
El Garbanzal, 16-IV-1973, R. Sagredo, H La Salle Almería (sub O. minor en Pujadas \& Lora, 1996). Base Sierra Lisbona, 30SWG9126, 209 m, 15-IV-2005, A. Pujadas, COA 33958. Sopalmo, Rambla del Mora, 30SWG9802, 16-IV-2005, A. Pujadas, COA 33957. Opp. Almería pagus Almadraba de Monteleva, 26-IV-1994, M. Tetera, PRC. CÁDIZ: Serranía de Ronda: Sierra Zafalgar, pagus Grazalema, 30-IV-1994, M. Tetera, PRC. Ubrique, Cerro de los Batanes, 19IV-1977, S. Silvestre, SEV 162942. Zahara de la Sierra, Arroyo Bocaleones, TF8577, 21-IV-1980, F. García et al., SEV 162983. CÓRDOBA: Sierra Morena: Espiel, Sierra de Espiel, 30S0321601/ 4226378, $612 \mathrm{~m}, 30-\mathrm{IV}-2007$, A. Pujadas \& E. Reyes Sepúlveda, COA 37646. GRANADA: Serranía de Ronda: Loja, Sierra de Loja, base Cerro de los Monjos, 30SUG9812, 800 m, 12-V1989, M.V. García et al., GDAC 33683. Sierras de Almijara, Guájares y Trevenque: Cogollos Vega, Peñón de la Mata, 30SVG5227, 1500 m, 22-V-1988, O. Socorro et al., GDA 22367. Depresión de Guadix-Baza y Guadiana Menor: Subida Cerro Jabalcón, 30SWG1858, 1300 m, 4VII-1998, F.B. Navarro Reyes, GDA 47772. Sierra Nevada y Filabres: Güejar-Sierra, inicio Vereda de la Estrella, 30SVG6212, 1050 m, 9VI-2000, A. Pujadas \& E. Triano, COA 33956. JAÉN: Subbética Occidental: Jaén, Puente de la Sierra, VG37, 600 m, 17-IV-1974, C. Fernández López, JAEN 741193 (sub O. amethystea en Molino Olmedo et al., 1995: 47). La Guardia de Jaén, VG37, 800 m, 1-VI-1975, C. Fernández López, JAEN 75700. Sierra Mágina: Campanario, 1200 m, 3-VII-1925, Cuatrecasas, BC 46694 (sub O. minor var. palestina en Cuatrecasas, 1929: 429). Versus Las Hermanas, 750 m, 4-VI-1926, Cuatrecasas, BC 46692 (sub O. minor en Cuatrecasas, 1929: 429). Bédmar, Barranco Perú, VG68, 740 m, 30-V-1980, C. Fernández López, JAEN 80644 (sub O.minor en Molino Olmedo et al., 1995: 49). Bélmez de la Moraleda, Sierra de la Cruz, 15-VII-1971, J. Fernández Casas, MA 412348. Cambil, La Mata, VG57, 1050 m, 18IV-1981, C. Fernández López, JAEN 81417 (sub O. alba en Molino Olmedo et al., 1995: 47). MÁLAGA: Sierra del Aljibe: Gaucín, carretera Gaucín-Algeciras, 30STF9043, 640 m, 24-IV2004, O. Gavira, MGC 57697. Serranía de Ronda: Antequera, E1 Torcal, 30SUF6290, 29-V-
1996, Y. Gil \& D. Navas, MGC 41840. Axarquía: In colle San Antón prope urbem Málaga, V-1845, H.M. Willkomm, COI-H ${ }^{\circ}$ Willkomm (sub $O$. minor $\gamma$ procerior; citado en Willkomm, 1870: 626). Vélez-Málaga, E1 Peñón del Toro, 30SVF0016, 17-IV-2004, J. García-Sánchez \& R. Torreblanca, MGC 64803.

Categoría UICN: LC

\section{O. hederae Duby, Bot. Gall. 350 (1828)}

CÁDIZ: Sierra del Aljibe: Tarifa, Sierra de Ojén, 30STE6799, 500 m, 22-V-1988, P. Garín, ARAN 34803. S ${ }^{a}$ La Palma-S ${ }^{\mathrm{a}}$ Luna, P.N. Los Alcornocales, Canuto de la Alcaria, 30STF6802, 20-VI-1995, A. Lora et al., COA 17469. Casa Huerto Campano, Venta de Ojén, Los Barrios, 30STF6701, 315 m, 26-IV-1993, C. Aedo et al., MA 526149. Jimena, TF7936, 26-V-1985, J. Cuenca \& L. Fernández, MGC 19315. Los Barrios, Sierra de Ojén, Chozas, El Pinillo, 4-VII1981, J. Arroyo et al., SEV 71824. Serranía de Ronda: Dehesa de la Jarilla, urbis Jerez [sic.], 28V-1878, Pérez Lara, MAF 36220 (sub O. minor). Algodonales, Sierra de Líjar, 31-V-1979, A. Aparicio et al., SEV 162964. Grazalema, Sierra del Pinar, 9-VII-1991, M. Arista, SEV 135002. CÓRDOBA: Sierra Morena: Trassierra, Arroyo Molino, Baños de Popea, 12-VII-2006, $A$. Pujadas \& P. Pujadas, COA 37500. Subbética Occidental: Rute, Sierra Horconera, 31-V-1987, C. Gálvez, COA 13489. Cabra, Cerro Camarena, 6-VI-1987, C. Gálvez, COA 17478. Cabra, La Nava, 6-VII-1995, A. Lora, COA 17665. GRANADA: Cazorla-Segura-Las Villas: Sierra de Castril, nacimiento, 30SWG2295, 1220 m, 7VII-1992, C. Salazar, GDAC 40018. Depresión de Granada: Cájar, 12-IV-1987, G. Guzmán, COA 13614. Sierras de Almijara, Guájares y Trevenque: Padul, Cerro de los Molinos, 30SVF4496, 750 m, 16-V-1989, Pérez Raya \& López Nieto, GDA 22035. La Alfaguara, Fuente de la Teja, 16-VI-1976, T. Palomeque, JAEN 630701. Sierra Nevada y Filabres: Laroles, 1200 m, 22-VI-1989, A. Hervás, GDAC 34718. GIBRALTAR: Sierra del Aljibe: In monte Peñón de Gibraltar, 1-VI-1881, Pérez Lara, MAF 36221. JAÉN: Sierra Morena: Andújar, R. De Villalba, 24-IV-1941, E. Guinea, MA 435417. Subbética Occidental: Sierra de Jabalcuz, 9-VI-1993, A. Pujadas et al., COA 17484. Jabalcuz, 
30SVG2878, 700 m, 18-V-1981, T. Palomeque, JAEN 832439 \& JAEN 832440. Los Villares, Río Frío, 30SVG2867, $1000 \mathrm{~m}, 6-\mathrm{VI}-1983, T$. Palomeque, JAEN 832442 (sub O. amethystea en Molino Olmedo et al., 1995: 47). Valdepeñas de Jaén, Cortijo Calderera, 30SVG2861, 1040 m, 7VII-1996, C. Fernández López, JAEN 960534 \& JAEN 960535. Garganta de los Ladrones, pr. Pantano de Quebrajano, 30SVG3566, $700 \mathrm{~m}$, Fernández Casas et al., 9-VI-1977, MA 409701. MÁLAGA: Sierra del Aljibe: Algeciras, Alcornocales, Arroyo de la Miel, 19-IV-2001, L. Plaza \& J. Salcedo, COA 31077. Cortes de la Frontera, Cañillas, 25-IV-2004, A. Pujadas \& L. Plaza, COA33981. Benarrabá, Arroyo de las Cobatillas, 30STF9746, $250 \mathrm{~m}, 29-\mathrm{V}-2004, O$. Gavira, MGC 61192. Genaguacil, Arroyo Aljaram, 30STF9744, $150 \mathrm{~m}, 6$-VI-2004, O. Gavira, MGC 61193. Jubrique, Rio Monardilla, 30SUF0148, 400 m, 19-VI-2004, O. Gavira, MGC 61186. Serranía de Ronda: Torcal de Antequera, 24-VII-1976, J. Guerra, GDAC 4443. Axarquía: Málaga, Jardín Botánico «La Concepción», 2-V-1995, B. Verdú \& T. Sánchez, COA 24865. SEVILLA: Sierra Morena: San Nicolás del Puerto, Arroyo Húmedo, 22-VI-1976, E.F. Galiano et al., MA 465689.

Categoría UICN: LC

Observaciones. No citada por Valdés (1987) para Andalucía Occidental.

\section{Especies no localizadas en Andalucía}

O. crinita Viv., Fl. Cors. Prodr.: 11 (1824). No existe ningún testimonio de herbario que respalde la presencia de $O$. crinita Viv. $[=O$. sanguinea f. crinita (Viv.) Beck] o de $O$. sanguinea C. Presl en Andalucía ni en toda la Península Ibérica (cf. Pujadas Salvà, 2002: 436437; Pujadas et al., 2003), por lo que las referencias de Beck (1890: 207; 1930: 299), Valdés (1987: 556), Pujadas Salvà et al., (1994: $135)$ probablemente correspondan a $O$. foetida var. broteri (Guim.) Merino (cf. Pujadas et al., 2003). Foley (2001a: 226; 2001b: 68-71) describe O. crinita var. occidentalis, sobre material gaditano, por confusión con $O$. foetida var. broteri (Guim.) Merino (cf. Pujadas et al., 2003: 740).

o. variegata Wallr., Orobanches Gen. Diask.: 40 (1825). No hemos podido encontrar ningún testimonio de herbario que respalde su presencia en Andalucía. En Pujadas Salvà et al. (1994: 135) ya se pone en duda la referencia de Sagredo (1987: 422) y las imágenes que aporta Kreutz (1995: 150-151) de Grazalema (Cádiz) pertenecen claramente a $O$. gracilis subsp. gracilis. De acuerdo con Foley (2001b: 72) $O$. variegata no se ha encontrado en toda la Península Ibérica.

O. caryophyllacea Sm. in Trans. Linn. Soc. London 4: 109 (1798). Citada por Willkomm (1870: 623), sub O. galii Vauch., en Sierra Nevada probablemente por confusión con $O$. clausonis Pomel. Las citas de Molino Olmedo et al. (1995: 48) son errores de identificación de especies pertenecientes a $O$. clausonis (JAEN 771222, JAEN 832441), a O. gracilis subsp. deludens (JAEN 81415, JAEN 4727) y a $O$. crenata (JAEN 832431, JAEN 832435).

No se encuentra en Andalucia ya que es de distribución más septentrional, del norte de la Península Ibérica, frecuentemente confundida con $O$. clausonis que también parasita a Rubiaceae (cf. Pujadas Salvà, 2001: 83; 2002: 416).

O. lutea Baumg., Enum. Stirp. Transsilv. 2: 215 (1816). No se ha encontrado en toda la Península Ibérica, su presencia en Andalucía es improbable. Se trata de una especie centroeuropea que quizá pueda llegar hasta el Pirineo (cf. Pujadas Salvà 2001: 79-80; Foley, 2001b: 71). Por lo tanto las citas para el teritorio andaluz hay que considerarlas como errores de identificación, así las referencias de Valdés \& Talavera (1991: 204-205) para la provincia de Jaén corresponden a $O$. haenseleri.

o. reticulata Wallr., Orobanches Gen. Diask. : 42 (1825). Diversos autores han citado esta especie en Andalucía (Willkomm, 1870: 621; Valdés, 1987: 552; Sagredo, 1987: 421, entre otros) probablemente confundida con $O$. crenata. Igualmente numerosos pliegos de herbario correspondientes a $O$. crenata han sido erróneamente identificados como O.reticulata (COFC 11764; JAEN 70163; JAEN 95802; JAEN 95823; JAEN 700163; JAEN 832445; JAEN 842553; JAEN 844111; JAEN 936058; JAEN 945489; MGC 16534; MGC 40474. SEV 31886; SEV 31888; SEV 31891; SEV 55031; SEV 71578; SEV 71579 SEV 110045; SEV 115473; 


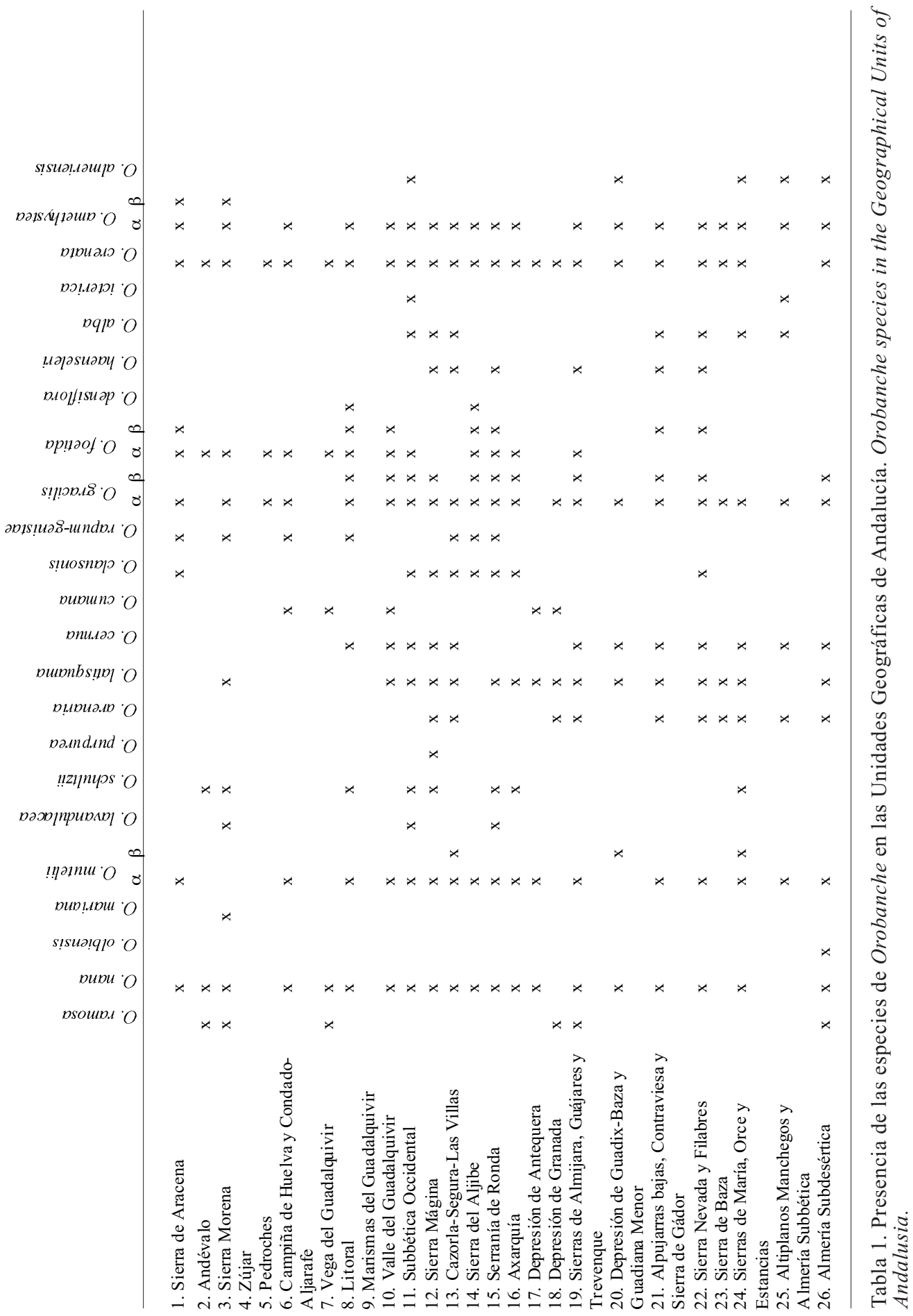


SEV 119955; SEV 119956; SEV 119957; SEV 120065; SEV 120066; SEV 120067; SEV 120068; SEV 120069; SEV 120720; SEV 138349; SEV 162957; SEV 162962) debido a la corola blanca con venas marcadas que le da un aspecto reticulado. En algunos casos se ha confundido con $O$. gracilis subsp. deludens (cf. Pujadas Salvà \& Plaza Arregui, 2004: 130).

Se han detectado algunos casos concretos, errores de identificación, como las referencias de Valdés \& Talavera (1991: 205) que corresponden a $O$. alba.

No hemos encontrado ningún testimonio de esta especie en todo el sur de la Península Ibérica, su localización se limita a las montañas del norte Peninsular (cf. Foley, 2001: 52; Pujadas Salvà, 2001: 82-83; 2002: 388).

o. artemisiae-campestris Vaucher ex Gaudin. Fl. Helv. 4: 179 (1829). Citada por Willkomm (1870: 624), sub O. loricata Reichenb., en Jaén, Cerro Zumbalejo (Lge), creemos que se trata de un error de identificación, posible confusión con $O$. minor, Willkomm (1870: 626) indica la presencia de O. minor también en Cerro Zumbalejo (Lge) y podría tratarse de la misma recolección. Las citas de Cuatrecasas (1929: 429), sub O. loricata Reichenb., de Campanil (BC 46677) corresponden a $O$. crenata Forsk.

Las citas de Pujadas Salvà et al., (1994: 135), sub O. loricata, son errores de identificación correspondientes a $O$. amethystea, O. crenata, O. minor. Las de Pujadas Salvà \& Lora González (1996: 214-215) de Almería son un error de identificación con $O$. amethystea. Las de Molino-Olmedo et al. (1995: 49) de Martos (JAEN 770675) y de Beas de Segura (JAEN 832019 ) corresponden a $O$. crenata y $O$. amethystea respectivamente.

Diversos pliegos de herbario, identificados como O. artemisiae-campestris u O. loricata Reichenb., corresponden a identificaciones erróneas de $O$. crenata (GDA 24458; GDAC 4443; JACA 215286; JAEN 751517; JAEN 77675; JAEN 970107; SALA 77937).

O. artemisiae-campestris se localiza en el noreste de la Península Ibérica, aunque su distribución es aún mal conocida (Pujadas Salvà, 2002: 398; Pujadas Salvà, 2007a).

Foley (2001b: 58) incluye $O$. santolinae en
O. artemisiae-campestris, además de $O$. picridis, sin una clara justificación, por lo que el tratamiento que se da en "Flora iberica" para este taxón es poco útil.

\section{Especie conflictiva que hay que buscar}

O. calendulae Pomel, Bull. Soc. Sci. Phys. Algérie 11: 110 (1874). Especie descrita como parásita de Calendula suffruticosa Vahl (Compositae). Se trata de un taxón conflictivo, algo dudoso, todos los ejemplares vistos bajo esta denominación han podido asignarse a otros taxones principalmente a $O$. amethystea y $O$. minor. Su presencia se ha señalado por error en Andalucía (Pujadas Salvà, 1994: 135).

Se requieren estudios más profundos para analizar la relación de $O$. calendulae con $O$. amethystea, $O$. minor y además con $O$. mauretanica Beck taxón con el que tradicionalmente se ha agrupado (Chater \& Webb, 1972: 291; Greuter et al., 1989: 257; Pujadas \& Lora,1995: 48; Pujadas Salvà, 2002: 401-402).

\section{CONCLUSIONES}

El género Orobanche está ampliamente distribuido por todo el territorio (tab. 1). Solamente ausente en dos unidades geográficas, Zújar - por ser un sector aislado y poco estudiado a nivel botánico- y Marismas del Guadalquivir -por tener unos ecosistemas poco propicios para su desarrollo.

Algunas especies son eurícoras, bien representadas en casi toda Andalucía, como $O$. crenata - presente en 23 unidades geográficas- ligada a la actividad humana principalmente agrícola, $O$. amethystea subsp. amethystea -en 19 unidades-, ligada a ambientes ruderalizados o subruderalizados, u O. gracilis subsp. gracilis -en 21 unidades-, presente en ecosistemas naturales o a veces subruderalizados. Otras son estenócoras presentes sólo en Andalucía Occidental - $O$. 
mariana presente en 1 sóla unidad geográfica, $O$. densiflora en 2 unidades, $O$. amethystea subsp. castellana en dos unidades o en Andalucía Oiental- $O$. olbiensis en 1 unidad, O. mutelii subsp. georgii-reuteri en 3 unidades, $O$. haenseleri en 6 unidades, $O$. alba en 7 unidades, $O$. almeriensis en 5 unidades. O. icterica presente en 2 unidades geográficas y $O$. lavandulacea en 3 unidades, estenócoras, se encuentran tanto en Andalucía Occidental como en Andalucía Oriental (tab. 1).

Las referencias para $O$. amethystea subsp. castellana y $O$. santolinae son las primeras citas contrastadas para toda Andalucía.

AGRADECIMIENTOS. A Carlos Aedo por facilitarnos los asientos corológicos que han servido para la redacción de "Flora iberica". A los conservadores y personal de los herbarios ABH, ALME, ARAN, BCN, CGE, COFC, COI, Colegio La Salle Almería, GDA/GDAC, Sánchez Pedraja, HUAL, JACA, JAEN, LEB, MA, MAF, MANCH, MGC, MPU, MUB, PRC, RNG, SALA/SALAF, SEV, VAL, W, WU, las facilidades en la consulta del material. A todos los colegas y amigos que han colaborado en la recolección de las plantas.

\section{BIBLIOGRAFÍA}

BECK, G. -1890- Monographie der Gattung Orobanche. Biblioth. Bot. 19: 1-275.

BECK, G. -1930- Orobanchaceae, in Engler (Ed.), Das Pflanzenreich 96 [IV. 261]: 1-348. Leipzig.

CABEZUDO, B., S. TALAVERA, G. BLANCA, C. SALAZAR, M. CUETO, B. VALDÉS, J.E. HERNÁNDEZ BERMEJO, C.M. HERRERA, C. RODRÍGUEZ HIRALDO y D. NAVAS -2005- Lista Roja de la Flora Vascular de Andalucía. Sevilla.

CHATER, A.O. \& D.A. WEBB -1972Orobanche, in Tutin, Heywood, Burges, Moore, Valentine, Walters \& Webb (Eds.),
Flora Europaea 3: 286-293. Cambridge

CUATRECASAS, J. -1929- Estudios sobre la flora y la vegetación del Macizo de Mágina. Treb. Mus. Ci. Nat. Barcelona, Ser. Bot. 12: $1-508$.

FOLEY, M.J.Y. -2001a- Orobanchaceae in the "Flora Iberica" area: new taxa, excluded taxa, and typification. Anales Jard. Bot. Madrid 58(2): 223-233.

FOLEY, M.J.Y. -2001b- Orobanche L., in: Paiva, Sales, Hedge, Aedo, Aldasoro, Castroviejo, Herrero \& Velayos (Eds.), Flora iberica 14: 32-72. Madrid.

GREUTER, W.R., H.M. BURDET \& G. LONG 1989- Med-Checklist. Vol. 4 (LauraceaeRhamnaceae). Genève.

IVORRA, A. - 2006-Flores de Almería. www.floresdealmeria.com (accesión el 15III-2007).

KREUTZ, C.A.J. -1995-Orobanche. The European broomrape species. Central and Northern Europe. Maastricht.

MOLERO MESA, J. y F. PÉREZ RAYA -1987La Flora de Sierra Nevada. Avance sobre el catálogo florístico nevadense. Granada.

MOLINO OLMEDO, F., A.J. PUJADAS SALVÀ, C. FERNÁNDEZ GARCÍA-ROJO y C. FERNÁNDEZ LÓPEZ - 1995- Orobanche de Jaén hasta 1993. Blancoana 12: 47-50.

PUJADAS, A. \& A. LORA -1995- Orobanche almeriensis A. Pujadas (Orobanchaceae), nueva especies del sureste de la Península Ibérica. Anales Jard. Bot. Madrid: 53(1): 4753.

PUJADAS, A. \& L. VELASCO -2000Comparative studies on Orobanche cernua L. and O. cumana Wallr. (Orobanchaceae) in the Iberian Peninsula. Bot. J. Linn. Soc. 134(4): 513-528.

PUJADAS SALVÀ, A.J. -1997- Orobanche ballotae A. Pujadas (Orobanchaceae) especie nueva. Acta Bot. Malacitana 22: 29-34.

PUJADAS SALVÀ, A.J. -1999- Orobanche icterica Pau, taxón minusvalorado del Sistema Ibérico. Fl. Montiber. 11: 15-18.

PUJADAS SALVÀ, A.J. -2000- Orobanche L. (Orobanchaceae) sect. Trionychon Wallr., en Andalucía. Acta Bot. Malacitana 25: 93-106.

PUJADAS SALVÀ, A.J. -2001- Aportació al coneixement del gènere Orobanche L. als 
Països Catalans. Orsis 16: 71-78.

PUJADAS SALVÀ, A.J. -2002- Orobanchaceae, in López Sáez, Catalán \& Sáez (eds.), Plantas Parásitas de la Península Ibérica e Islas Baleares 345-451. Mundi Prensa, Madrid.

PUJADAS SALVÀ, A.J. -2006- Nuevas combinaciones nomenclaturales y aportaciones florísticas en el género Orobanche L. (Orobanchaceae). Lagascalia 26: 246-248.

PUJADAS SALVÀ, A.J. -2007a- Orobanche, in Gómez (Ed.), Atlas de la Flora de Aragón. www.ipe.csic.es/floragon (accesión el 4-II2007).

PUJADAS SALVÀ, A.J. -2007b- Orobanche mariana A. Pujadas (Orobanchaceae) sp. nov. para el sur de la Península Ibérica. Acta Bot. Malacitana 32: 269-274.

PUJADAS SALVÀ, A.J. -2007c- Novedades taxonómicas y nomenclaturales en el género Orobanche (Orobanchaceae). Acta Bot. Malacitana 32: 265-267.

PUJADAS SALVÀ, A.J., P. FRAGA I ARGUIMBAU, E. SÁNCHEZ GULLÓN \& N. MOLINA MAHEDERO-2003-Orobanche crinita and Orobanche foetida (Orobanchaceae) in the West Mediterranean area. Bocconea 16(2): 737-744.

PUJADAS SALVÀ, A.J., E. HERNÁNDEZ BERMEJO \& J.A.R. OLIVEIRA VELLOSO -1994- The genus Orobanche in Andalusia (southern Spain); taxonomical, chorogical and ecological aspects, in Pieterse, Verleij \& ter Borg (Eds.), Biology and management of Orobanche, Proceedings of the Third International Workshop on Orobanche and related Striga research, 132-137. Amsterdam.

PUJADAS SALVÀ, A.J. \& Á. LORA GONZÁLEZ - 1996- El género Orobanche L. (Orobanchaceae) en la provincia de Almería, SE de España. Acta Bot. Malacitana 21: 199220.

PUJADAS SALVÀ, A.J. y Á. LORA GONZÁLEZ -1997- Distribución de Orobanche clausonis Pomel (Orobanchaceae) en la Península Ibérica y Baleares. Anales Jard. Bot. Madrid 53(2): 477-479.
PUJADAS SALVÀ, A.J. \& L. PLAZA ARREGUI -2004- What is Orobanche haenseleri var. deludens Beck?. Anales Jard. Bot. Madrid 61(2): 129-134.

PUJADAS SALVÀ, A.J., L. PLAZA ARREGUI, E. TRIANO MUÑOZ y J. ALGARRA ÁVILA -2005- Orobanche icterica (Orobanchaceae) revisited. Bot. J. Linn. Soc. 148: 117-124.

PUJADAS SALVÀ, A.J., D. RUBIALES OLMEDO y M. LÓPEZ MARTÍNEZ -2005Orobanche L. (Orobanchaceae) sect. Trionychon Wallr., en Andalucía II: Orobanche rosmarina Beck. Acta Bot. Malacitana 30: 49-54.

SAGREDO, R. -1987- Flora de Almería. Almería.

SÁNCHEZ GULLÓN, E., F.J. MACÍAS, P. WEICKERT y B. VALDÉS -2005Aportación a la Flora y Vegetación de la cuenca baja del Río Guadiana en el Andévalo Occidental (España). Lagascalia 25: 252-257.

SCHNEEWEISS, G.M, T. PALOMEQUE, A.E. COLWELL \& H. WEISS-SCHNEEWEISS 2004- Chromosome numbers and karyotype evolution in holoparasitic Orobanche (Orobanchaceae) and related genera. Am. J. Bot. 91: 439-448 \& Supl. Data: 1-10.

UICN -2001- Categorías de las Listas Rojas de la UICN: Versión 3.1. Comisión de la Supervivencia de las Especies de la UICN. Gland \& Cambridge.

VALDÉS, B. -1987- Orobanchaceae, in Valdés, Talavera \& Fernández Galiano (Eds.), Flora Vascular de Andalucía Occidental 2: 550-558. Barcelona.

VALDÉS, B. -2005- Nuevas plantas y nuevas áreas para la Flora de Andalucía y $\mathrm{N}$ de Marruecos. Lagascalia 25: 193-204.

VALDÉS, B. \& S. TALAVERA -1991- Checklist of the vascular plants collected during Iter Mediterraneum I. Bocconea 1: 43-286.

WILLKOMM, H.M. -1870- Orobanchaceae, in: Willkomm \& Lange (Eds.), Prodromus Florae Hispanicae 2: 620-632. Stuttgart.

WILLKOMM, H. M. -1893- Supplementum prodromi florae hispanicae. Stuttgart. 